\title{
GASEOUS RADIONUCLIDE ACTIVITY IN THE BUILDING 6010 EXHAUST DETERMINED BY GAMMA-RAY ASSAY OF CRYOGENIC LIQUIFIED SAMPLES
}

\author{
J. K. Dickens
}

DATE PUBLISHED - January 1991

Prepared for the Office of Energy Research Division of Nuclear Physics

Prepared by the

OAK RIDGE NATIONAL LABORATORY

Oak Ridge, Tennessee 37831 managed by

MARTIN MARIETTA ENERGY SYSTEMS, INC.

for the

U.S. DEPrRTMENT OF ENERGY

under contract DE-AC05-84OR21400 


\section{CONTENTS}

ACKNOWLEDGMENTS . . . . . . . . . . . . . . . vii ABSTRACT . . . . . . . . . . . . . . . . . . . ix

1. INTRODUCTION

2. EXHAUST-STACK SAMPLES, MEASUREMENTS, AND ORELA CONDITIONS . . . . . . . . . . . . . . . . . . 6

3. GAMMA-RAY DETECTOR CALIBRATION . . . . . . . . . 8

4. GAMMA-RAY DATA REDUCTION . . . . . . . . . . . . . 11

5. ANALYSIS OF THE REDUCED GAMMA-RAY DATA . . . . . . 13

$5.1 \mathrm{FOR}^{41} \mathrm{Ar}$. . . . . . . . . . . . . . . . . . 13

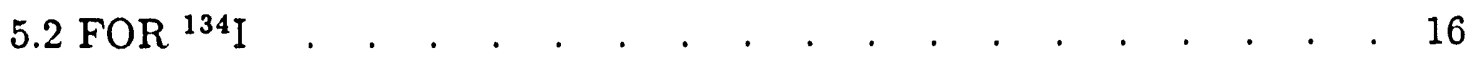

5.3 FOR MASS $=133$ ISOTOPES . . . . . . . . . . . . . . . . 19

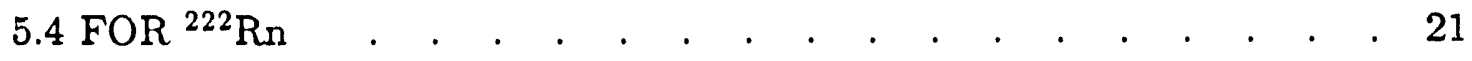

5.5 FOR ${ }^{13} \mathrm{~N}$ AND ${ }^{11} \mathrm{C}$. . . . . . . . . . . . . . . . . . 23

5.6 OTHER RADIONUCLIDES . . . . . . . . . . . . . . . . . . 29

6. SUMMARY AND CONCLUSIONS . . . . . . . . . . . . 30

REFERENCES . . . . . . . . . . . . . . . . . . 32 


\section{LIST OF FIGURES}

Fig. $\quad \underline{\text { Page }}$

1 Cross-sectional view of the Marinelli counting dewar . . . . . 5

2 Measured efficiencies of the Ge detector used in this study . . . 9

3 Comparison of the present adopted efficiency function with adjusted efficiencies of similar detector as given in ref. $9 . \quad . \quad . \quad . \quad . \quad . \quad$. 10

4 Raw spectral data corresponding to the gamma-ray energy region including the $E_{\gamma}=1294 \mathrm{keV}$ gamma ray following decay of ${ }^{41} \mathrm{Ar} \quad . \quad 14$

5 Results of measuring ${ }^{41} \mathrm{Ar}$ decay . . . . . . . . . . . . 15

6 Relevant portion of the spectrum obtained by summing runs 27 to 30 for the analysis of the presence of ${ }^{134} \mathrm{I}$ in Sample No. 1 . . . . 18

7 Relevant portions of the final spectrum for the analysis of the presence of ${ }^{133 \mathrm{~m}} \mathrm{Xe}$ and ${ }^{133} \mathrm{I}$. . . . . . . . . . . . . . . 20

8 Relevant portions of three spectra for the analysis of the presence of ${ }^{222} \mathrm{Rn}$ in the sample

9 Counting rate for the peak corresponding to detection of annihilation radiation as a function of the time following the end of sample collection 


\section{ACKNOWLEDGMENTS}

I express my appreciation to C. D. Phillips and his crew from the Systems and Equipment Department for efficient and timely sample collections and to T. A. Lewis and D. C. Larson for illuminating discussions as the initial, preliminary results from the gamma-ray assay measurements became available. My thanks go also to R. W. Peelle who provided encouragement, support, and a thorough reading of the draft manuscript. I also thank S. R. Damewood for careful manuscript preparation. 


\begin{abstract}
Samples of gaseous components in the exhaust stack of Building 6010 at the Oak Ridge National Laboratory were obtained for two conditions, (a) the Oak Ridge Electron Linear Accelerator in a normal operating mode, and (b) the accelerator shut down. The decay of one radionuclide, ${ }^{222} \mathrm{Rn}$, was observed equally in both measurements. The decay of three radionuclides, namely ${ }^{11} \mathrm{C},{ }^{13} \mathrm{~N}$ and ${ }^{41} \mathrm{Ar}$, was observed during accelerator operation but not during shutdown. Gamma-ray assay measurements were obtained using a calibrated, high-resolution, Ge detector system. Background data were obtained to ascertain quantitatively the sampleindependent contributions to the measurements. Data reduction utilized a combination of computer and manual methods. A complete analysis was carried out to determine the actual measured isotope radioactivity density (in $\mathrm{pCi} / \ell$ ) for the particular conditions existing at the time the samples were collected. Corrections were applied to these resulis to account for non-constant sample collection rates and for sample transfer losses. A complete report of all facets of the experiment is given.
\end{abstract}




\section{INTRODUCTION}

On Friday, June 2, 1989, personnel from the Systems and Equipment Department of the Quality and Technical Services Division at the Oak Ridge Gaseous Diffusion Plant (now known officially as Oak Ridge $K-25$ Site) performed a cryogenic sampling of the exhaust flowing out the exterior stack of Building 6010. Air from the entire building including hoods and (as discussed below) the target room is transmitted from the building through this stack located south of the building. The top of this exhaust is $52.5 \mathrm{ft}$ above the ground. Gamma-ray measurement techniques were used to determine the presence of gaseous radionuclides in the building's exhaust during operation of the Oak Ridge Electron Linear Accelerator (ORELA). They reported ${ }^{1}$ positive findings of five radioisotopes, namely ${ }^{41} \mathrm{Ar},{ }^{133} \mathrm{I},{ }^{133 m} \mathrm{Xe}$, ${ }^{134} \mathrm{I}$, and ${ }^{222} \mathrm{Rn}$. The quantitative values given in their report indicated that the volume amounts of each and every one of the five radionuclides was well below the Derived Air Concentration (DAC) for Workers from External Exposure During Immersion in a Contaminated Atmospheric Cloud as given in the Department of Energy (DOE) Order $5480.11 .^{2}$ The presence of ${ }^{41} \mathrm{Ar}$ could be understood as being a product of neut on interactions with argon in the air (the neutrons being produced during accelerator operation), and the presence of ${ }^{222} \mathrm{Rn}$ was expected since this radioisotope is present in the atmosphere. ${ }^{3}$ The identification of the remaining three radionuclides, ${ }^{133} \mathrm{I}_{2}{ }^{133 m} \mathrm{Xe}$, and ${ }^{134} \mathrm{I}$, however, was a surprise; none of these isotopes should be generated in the normal course of ORELA operation.

As the facility name implies, the ORELA is a linear accelerator of electrons. At the end of the acceleration phase the electrons have obtained a nominal energy of $\sim 140 \mathrm{MeV}$. The machine operates in a burst mode and is capable of providing up to 1000 bursts/second. The burst width is a parameter of operation and can be set to be between about 3 and $25 \mathrm{nsec}$. The instantaneous electron power is also a parameter of operation and has a nominal maximum of $\sim 2$ joules $/ \mathrm{nsec}$. The maximum power available from the accelerator is 50 kilowatts.

After the electron beam leaves the accelerator, it is guided into an area designated as the "target" room. This room is a very well shielded room of approximately $1700 \mathrm{cu}$. ft interior volume; during accelerator operation the pressure in this room is maintained between 20 and $30 \mathrm{~mm} \mathrm{Hg}$ by a vacuum pump. The exhaust from this pump is fed directly into the building's exhuust stack and so is sarnpled by the cryogenic sampling methods discussed below. In the target room one may choose between two different target configurations. The one used most of the time consists of a target made of tantalum strips and cooled by water flowing around and between the strips. When the electron beam hits this target, much of the incident energy is converted into bremsstrahlung (photon) radiation, and then this radiation subsequently interacts with the tantaium to produce neutrons via photonuclear reactions. Some of these neutrons are moderated (i.e., slowed down) by subsequent interactions in the tantalum and in the water surrounding the tantalum. Emanating from the target, then, is a "white spectrum" of neutrons, ${ }^{4}$ in other words, neutrons are created having all energies between the very wide limits of thermal and $\sim 100 \mathrm{MeV}$. The second target configuration consists of two parts: the first part is a relatively small, water cooled, cup made of tantalum which acts as a converter by interacting with the incident electrons to form the bremsstrahlung radiation; the bremsstrahlung is then intercepted by a target made of beryllium, and neutrons 


\section{INTRODUCTION}

are created by photonuclear reactions. ${ }^{5}$ Again, a "white spectrum" of neutrons is created; however, this mode of operation is used principally for those experiments requiring neutrons having high energies, particularly $E_{n}>10 \mathrm{MeV}$.

It was deemed desirable to repeat the study of the possible presence of gaseous radionuclides in the building's exhaust for several reasons. Firstly, the reported ${ }^{1}$ presence of the radionuclides of I and Xe needed further study since the mechanism for their creation by the normal operation of the accelerator was not apparent. Secondly, the earlier sampling had occurred during accelerator operation in the second mode described above; it seemed desirable to obtain a sample not only for the more usual mode but also to obtain a sample when the accelerator was not operating. Thirdly, the counting of the sample by personnel of the Quality and Technical Services Division of IK-25 did not commence until their return to K-25, or a period of several hours following the completion of sample collection; as a consequence any short-lived gaseous radionuclide would not have been observed. In particular, the production of the radionuclide ${ }^{13} \mathrm{~N}$ during accelerator operation was anticipated from neutrc: and photon interactions with the nitrogen in the air. The isotope ${ }^{13} \mathrm{~N}$ has a 10 -minute half life. ${ }^{6}$ Therefore, it was decided to do the gammaray assay portion of the sampling using gamma-ray counting equipment in Building 6010 so as to be able to initiate the gamma-ray assay of the collected sample soon enough to detect gamma radiation signifying detection of decay of ${ }^{13} \mathrm{~N}$.

The total volume of air in the target room reduced to a pressure of $1 / 30^{\text {th }}$ of an atmosphere is still rather substantial, and one may ask if there are additional radionuclides that could be created by neutron interactions with constituents of the residual air in the target room. Table 1 presents the nominal amounts of various gases in a "standard" dry atmosphere ${ }^{3}$. One may note that the "expected" radionuclides discussed above, namely ${ }^{13} \mathrm{~N}$ and ${ }^{41} \mathrm{Ar}$ can be produced from two of the three major constituents. In addition, a value is given for the concentration of racion in the atmosphere. If this value represented entirely ${ }^{222} \mathrm{Rn}$ (likely not a poor approximation) then one may calculate that this value corresponds to about $8.7 \mathrm{pCi} / \ell$ which, in value, may be compared with the maximum $\mathrm{DAC}$ allowed of 80 $\mathrm{pCi} / \ell$ in DOE Order 5480.11 . $^{2}$ The only other radionuclides that might be anticipated are ${ }^{15} \mathrm{O}$ from neutron interactions with oxygen having a half life of $122 \mathrm{sec}$, and ${ }^{11} \mathrm{C}$ having a half life of $20.3 \mathrm{~min}$ from neutron interactions with any carbon in the atmosphere and on inside surfaces of the target room or surfaces of target support mechanisms. Detection and identification of ${ }^{15} \mathrm{O}$ was exp zcted to be difficult because of its short lifetime. Identification of the longer-lived ${ }^{11} \mathrm{C}$ isotope was feasible, but initially it was anticipated that not very much of this isotope would be created. However, as presented later on, the presence of ${ }^{11} \mathrm{C}$ was identified at a radioactivity density of $\sim 25 \%$ that of ${ }^{13} \mathrm{~N}$. Detection of all three of these lightelement radioisotopes, ${ }^{11} \mathrm{C},{ }^{13} \mathrm{~N}$, and ${ }^{15} \mathrm{O}$, utilized measuring the $\beta^{+}$annihilacion radiation, $E_{\gamma}=511 \mathrm{keV}$, since none of these three radionuclides has any other gamma radiations following decay. Thus, the only way to identify the responsible radionuclide in the measurements of the $511-\mathrm{keV}$ data would be by a careful study of the peak yields (in the spectral dara) as a function of the time following the end of the collection of the cryogenic sample. 
Table 1. Sea-level Atmospheric Composition for a Dry Atmosphere ${ }^{a, b}$

\begin{tabular}{lc}
\hline Constituent Gas & $\begin{array}{c}\text { Mol. Fraction } \\
(\%)\end{array}$ \\
\hline Nitrogen $\left(\mathrm{N}_{2}\right)$ & 78.09 \\
Oxygen $\left(\mathrm{O}_{2}\right)$ & 20.95 \\
Argon $(\mathrm{A})$ & 0.93 \\
Carbon dioxide $\left(\mathrm{CO}_{2}\right)$ & 0.03 \\
Neon $(\mathrm{Ne})$ & $1.8 \times 10^{-3}$ \\
Helium $(\mathrm{He})$ & $5.24 \times 10^{-4}$ \\
Krypton $(\mathrm{Kr})$ & $1.0 \times 10^{-4}$ \\
Hydrogen $\left(\mathrm{H}_{2}\right)$ & $5.0 \times 10^{-5}$ \\
Xenon $(\mathrm{Xe})$ & $8.0 \times 10^{-6}$ \\
Ozone $\left(\mathrm{O}_{3}\right)$ & $1.0 \times 10^{-6}$ \\
Radon $(\mathrm{Rn})$ & $6.0 \times 10^{-18}$ \\
\hline
\end{tabular}

${ }^{a}$ Taken from ref. 3 .

${ }^{b}$ These values are taken as standard and do not necessarily indicate the exact condition of the atmosphere. Ozone and radon particularly are known to vary at sea level and above. Traces of methane $\left(\mathrm{CH}_{4}\right)$ are also found, especially near marsh areas.

The basic cryogenic sampling technique has been described in a patent application by Perdue and Haywood. ${ }^{7}$ Briefly, the system consists of a steel sampling container of several liters $(\ell)$ liquid volume immersed in a liquid nitrogen bath so as to cool the container completely to the temperature of the boiling point of liquid nitrogen. A short metal probe is inserted into the exhaust stack, and the extracted yas is transmitted $\sim 50 \mathrm{~cm}$ in flexible tygon tubing to a particulate filter and a fowmeter. The gas then enters the neck of the sampling container through a small orifice. As part of the set-up procedure, a small amount of liquid nitrogen is placed inside the sampling container, and then the orifice holder is inserted into the neck of the sampling container, thence to the tubing from the filter and flowmeter. The air inside the sampling container begins to liquefy since its boiling point is several degrees higher than that of the liquid nitrogen. ${ }^{8}$ Following the liquefying of this air the pressure inside the sampling container becomes negative with respect to the gas in the ubing and so the gas in the tubing is drawn into the sampling container. The rate at which the outside gas is drawn into the sampling container depends upon the volume in the container which has not yet been occupied by the liquid sample. Thus, the rate of sampling slowly decreases as the sampling container fills up with liquified sample.

For relatively long-lived radionuclides in the sample the non-constant rate of collection is not important, but for several of the radionuclides observed in the present experiment it is necessary to estimate the rate of volume collection as a function of the time of the collection. The total time required to collect each sample in this experiment was $105 \mathrm{~min}$. The total volume, $V$, of exhaust gases for each 


\section{INTRONUCTION}

sample was $362.9 \ell$. The total time of collection, $T$, was 105 minutes. The average collection rate, then, was

$$
C_{\mathrm{av}}=V / T=3.46 \ell / \mathrm{min}
$$

The initial rate of gas collection was $\sim 4.2 \ell / \mathrm{min}$, and the final rate was $\sim 2.8 \ell / \mathrm{min}$. For the purposes of the computations in the following analyses of the measurements, it was assumed that the collection rate followed an exponential law, namely,

$$
R(t)=R_{i} \times e^{-\alpha t}
$$

where $R_{i}$ was the initial rate of gas volume collection. An important boundary condition on $\alpha$ was the total volume, $V$ :

$$
V=R_{i} \times\left(1-e^{-\alpha T}\right) / \alpha,
$$

The volume, $V$, was the only exact number available, the $R_{i}$ being only approximately given as $4.2 \mathrm{\ell} / \mathrm{min}$. The solution for $\alpha$ is, therefore, somewhat imprecise; for the present purpose, however, a satisfactory value for $\alpha$, namely,

$$
\alpha=6.78 \times 10^{-5} / \mathrm{sec}
$$

was determined from Eqs. (1) and (2), and it was used in the analyses of the data as discussed below.

The volume of cryogenic liquid collected in each case was $\approx 0.6 \ell$. Following cessation of sample collection, the liquid sample was transferred from the sampling container into a pre-cooled Marinelli beaker of the type shown in Fig. 1. As observed in this figure, the particular beaker used is also a dewar. Measurements have been reported ${ }^{9}$ which determined the amount of loss of the liquified sample during this transfer. An average value of (5.5 \pm 0.7$) \%$ loss was deduced from these measurements. This loss factor was included in the final analyses of the present data.

After the sample transfer the Marinelli beaker was filled to a predetermined level with ordinary liquid nitrogen, a small rubber stopper was inserted into the neck of the beaker (primarily to keep out moisture), and then the beaker was transported quickly to the laboratory for gamma ray assay. The liquid sample evaporates slowly enough not to affect the potential measurement for the $10-\mathrm{min}$ isotope ${ }^{13} \mathrm{~N}$, but it could affect measurements of the longer-lived isotopes. However, as the liquid evaporates an effective elemental separation does occur. In particular, the concentration of oxygen increases which is of some concern because the increase could pr vage a potentially explosive atmosphere. ${ }^{9}$ One might also expect that the liquified $x$ adon would remain in the sample since its boiling point is substantially higher than the boiling point of liquid nitrogen.

Two cryogenic samples were extracted from the building's exhaust on Friday, August 24, 1990, one with the ORELA operating and the other with the ORELA shut down and the target-room pump turned off and isolated trom the building's exhaust stack. Details of the measurement conditions, the presently-used gammaray measuring system, the gamma-ray data accumulation, and the data reduction are given in the next sections. 
ORNL. OWG $80-18661$

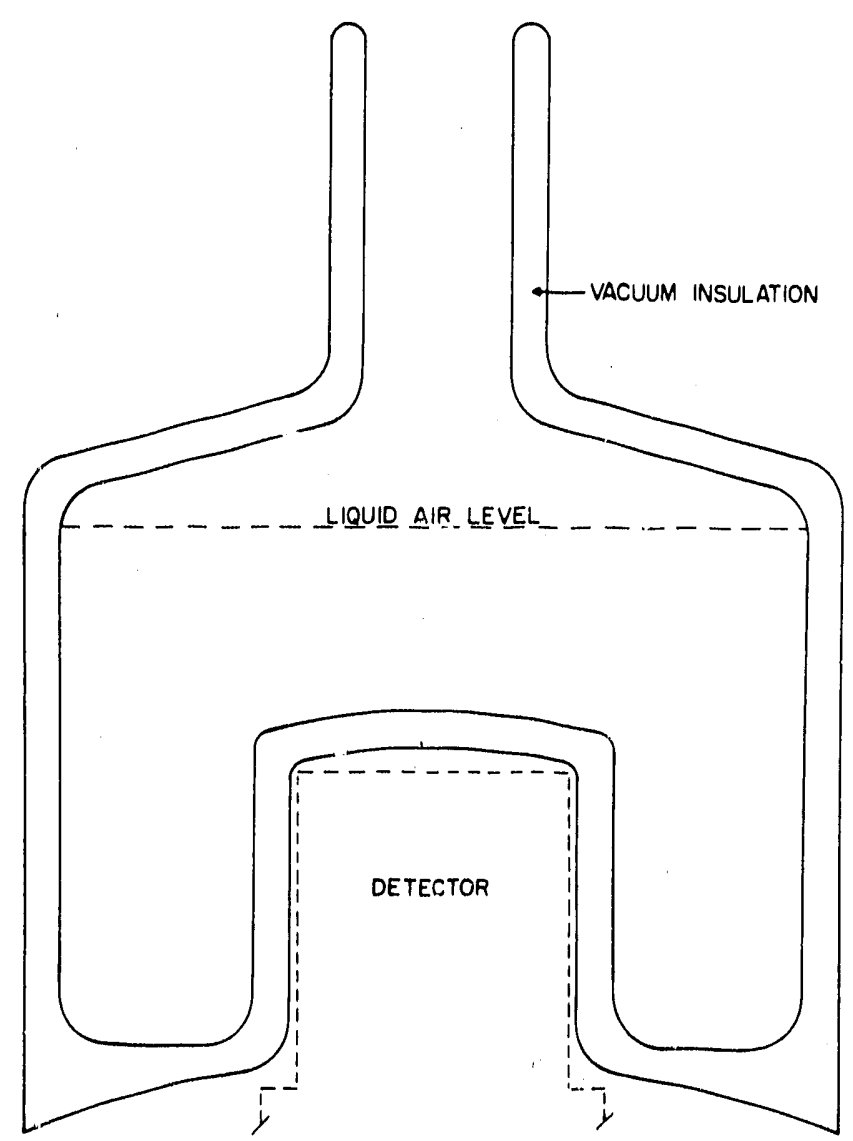

Fig. 1. Cross-sectional view of the Marinelli counting dewar. 


\section{EXHAUST-STACK SAMPLES, MEASUREMENTS, ANJD ORELA CONDITIONS}

A total of three samples in the Marinelli beakers were studied with the $\mathrm{Ge}(\mathrm{Li})$ detection system. The three samples were:

Sample No. 1: Exhaust stack with the ORELA operating and the exhaust from the ORELA target room included in the building exhaust.

Sample No. 2: Exhaust stack with the ORELA shut off and the exhaust from the ORELA target room excluded from the building exhaust.

Sample No. 3: A "blank" used to determine the room background recorded in the partially shielded gamma-ray detection system.

Sample No. 1 was obtained between 9:15 and 11:00 am EDT on 24 August 1990. The ORELA was operating at about 15 kilowatts of power, the tantalum target was in use, and the partial pressure of the target room was about $25 \mathrm{~mm} \mathrm{Hg}$ (or about 1/30 of atmospheric pressure). At 11:10 am the ORELA was shut down, and about 10 minutes later the target room vacuum pump was shut down and its valve was closed. Sample No. 2 was obtained between 11:45 am and 1:30 pm EDT on the same day. At about 5:00 pm, after several spectra were obtained, Sample No. 2 was discarded. Sample No. 3 was prepared in the now empty Marinelli beaker by filling it with "virgin" liquid nitrogen from the Bldg. 6010 supply. The reasons for obtaining the background radiation using Sample No. 3 were (a) the observation that the sample liquid, surrounding the $\mathrm{Ge}(\mathrm{Li})$ detector as it does, acted as a shield thus reducing the "room background" activity in the detector during the sample measurements, and (b) the opposite effect that could be observed if ordinary liquid nitrogen, which, it will be recalled from above, was used to normalize the volumes of Sample No. 1 and Sample No. 2 in their Marinelli beakers, may have had any small but measurable residual radioactivity in itself.

The gamma-ray assay measurements were initiated as quickly after the end of each of the two collection periods as possible. Table 2 su amarizes the gamma-ray measurements that were made. The measurements were completed within 13 hours. At this point in time Sample No. 1 was discarded so as to avoid the unlikely but potential difficulty due to the oxygen enrichment problem discussed above.

As indicated in Table 2, eleven gamma-ray spectra were obtained; the analyzer and electronics were set to record 4096 channels of pulse-height data at a dispersion of $0.70 \mathrm{keV}$ per channel. These settings were chosen so that gamma radiation from all anticipated potential gaseous radionuclides and all known background radionuclides could be observed if present in sufficient quantity in the exhaust-stack sample. The choices of waiting times and counting times were predicated on the $a$ priori desire to obtain information on the possible production of $10-\mathrm{min}^{13} \mathrm{~N}$ and/or $1.8-\mathrm{hr}{ }^{41} \mathrm{Ar}$ and any other gaseous radioisotope whose creation could be ascribed to accelerator operation. 
Table 2. Summary of gamma-ray assay measurements.

\begin{tabular}{lccc}
$\begin{array}{c}\text { Run } \\
\text { No. }\end{array}$ & $\begin{array}{c}\text { Sample } \\
\text { No. }\end{array}$ & $\begin{array}{c}T_{w}^{a} \\
\text { (sec) }\end{array}$ & $\begin{array}{c}T_{\text {count }}{ }^{b} \\
\text { (sec) }\end{array}$ \\
\hline 27 & 1 & 300 & 600 \\
28 & 1 & 900 & 623 \\
2.9 & 1 & 1540 & 800 \\
30 & 1 & 2340 & 800 \\
31 & 1 & 4320 & 2500 \\
32 & 2 & 600 & 700 \\
33 & 2 & 1320 & 1250 \\
34 & 1 & 11700 & 3000 \\
35 & 2 & 8280 & 3613 \\
36 & 3 & 26100 & 3608 \\
37 & 1 & 14900 \\
\hline Time after the end of the sample collection period. \\
${ }^{b}$ Gamma-ray-spectrum accumulation period.
\end{tabular}




\section{GAMMA-RAY DETECTOR CALIBRATION}

The gamma-ray detector is a $\mathrm{Ge}(\mathrm{Li})$ detector of nominally $2.0-\mathrm{keV}$ resolution for detection of $1.23-\mathrm{MeV}$ gamma rays. It is classifie $\mathrm{i}$ as a "15\%" detector, meaning that its "efficiency" for detection of the $1.33-\mathrm{MeV}$ gamma ray is $15 \%$ of the detectior efficiency of that ganma ray using a 3-in. by 3-in. NaI cylindrical detector at a scurce-to-detector distance of $25 \mathrm{~cm}$. This $\mathrm{Ge}(\mathrm{Li})$ detector is normally maintained in a lead housing which completely surrounds and shields the detector. The system is ho. sseci in Lab E of Building 6010. The Marinelli beakers of the present experiment. however, are too large to fit into the top opening of the lead shielding, and so the entir'e top of the shielding was removed. As a consequence the room background radiation incieused substantially (about a factor of five), and this background includes gamm? rays identified with decay of ${ }^{222} \mathrm{Rn}$ which is one of the radioactive gases to be quantified in the exhaust-stack samples. This background was measured and is included in the analysis of the data presented in the next section.

Efficiency calibratin! of the $\mathrm{Ge}(\mathrm{Li})$ detector utilized two standard solutions prepared $^{10}$ in Marinelli beakers similar to those used to contain the exhaust-stack samples. Une solution was prepared from a standard ubtained from AmershamSearle ${ }^{11}$ containing the radioisotopes ${ }^{109} \mathrm{Cd},{ }^{57} \mathrm{Co},{ }^{113} \mathrm{Sn},{ }^{137} \mathrm{Cs},{ }^{88} \mathrm{Y}$ and ${ }^{60} \mathrm{Co}$. The standard was $\sim 2$ years ild, sc lifetime coriections for several of these radioisotopes were rather large. "The other c jution was prepared from a standard obtained from the U.S. National Bureau of Standards (NBS) ${ }^{12}$ containing the radionuclides ${ }^{125} \mathrm{Sb}$, ${ }^{154} \mathrm{Eu}$ and ${ }^{155} \mathrm{Eu}$. This standard was $\sim 7$ years o!d, and lifetime corrections were in :orporated for all three radionuclides. Each calibration measurement required about. a day, and they were completed before the exhaust-stack samples were drawn.

The measured spectral data were manually analyzed to determine the efficiency of the detection system for each gamma ray. The results of this experiment and analysis are given in Fig. 2. Somewhat disconcerting was the $\sim 8 \%$ disagreement between the two standards for $E_{\gamma}$ between about 0.2 and $1.0 \mathrm{MeV}$. This author's experience with standards prepared by Amersham Searle over several decades has heen extensive and very pnsitive. Consequersily, the aciopted efficiency function (peak effiriency vs gamma-ray energy), given by the solid line in Fig. 2, favors the ufficiencies determined from the Amersham Searle standard. For $E_{\gamma}=1294$ $\mathrm{keV}$ (i.e., the gamma-ray energy associated with ${ }^{41}$ Ar decay) the efficiency function agrees with both standards; however, for $E_{\gamma}=511 \mathrm{keV}$ (principally ${ }^{13} \mathrm{~N}$ decay) the measured yields (discussed below) would be $0 \%$ larger if the efficiency had been determined solely from the trend of efficiency indicated by the NBS standard. In paising, it shcild be noted that the standard solutions are water based with a specific gravity of 1.1$) \mathrm{gm}_{\lrcorner} / \mathrm{cc}$, whereas the cryogenic samples are liquid-nitrogen based with a specific gravity of $0.81 \mathrm{gm} / \mathrm{cc}$. Thus, there will be energy-dependent differences in the efficiencies exhibited in Fig. 2 and the efficiencies of gamma-ray detection in the liquid-niirogen medium due to the fact that the latter will not attenuate gamma radiation as much as the water. The differences in efficiencies are, however, very difficult to calculate because of the extencied geometry of the Marinelli beaker. An estinnate for $E_{\gamma}=0.2 \mathrm{MeV}$ indicates that the liquid $\mathrm{N}_{2}$ efficiency should be $=7 \%$ larger than the efficiency determined from the standards measurements; this up tions were made for this effect; indeed, had the adopted efficiency function shown 
ORNL-DWG 90N1-15754R

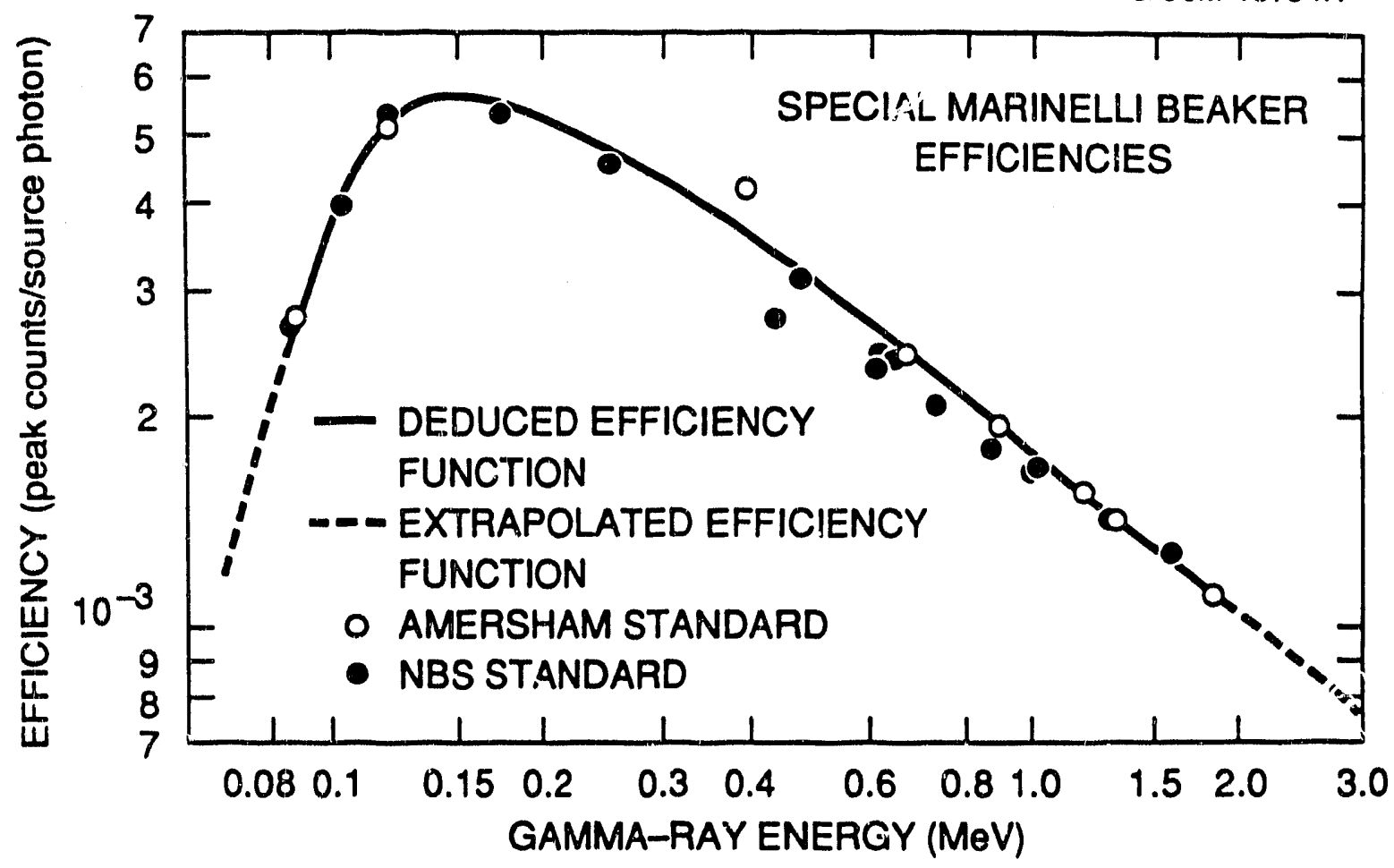

Fig. 2. Measured effleiencies of the Ge detector used in this study. The deduced efficiency as a function of $E_{\gamma}$ is indicated by the solid line. Extrapolated efficiencies are indicated by the dashed lines; however, the only gamma ray observed in the regions of $E_{\gamma}$ covered $h_{v}$ the dashed lines was the $2.61-\mathrm{MeV}$ radiation due to the presence of ${ }^{228} \mathrm{Th}$ in the room background. The adopted efficiency function is made up of deduced efficiency plus the extrapolated efficiency.

in Fig. 2 been developed by taking an average of the results for both standards, and then corrected for some estimates of differences due to the differences in the media, the final efficiency function would be very little different from the adonted function exhibited in Fig. 2.

As a quick check on the present efficiency function determination, it was compared with measured efficiencies reported for a "10\%" $\mathrm{Ge}(\mathrm{Li})$ system" obtained using the same two standards (in the same Marinelli beakers). This comparison is shown in Fig. 3, in which the previous (10\%) values have been multiplied by 1.5 to account for the approximate increase of efficiency for the present detector. Again, the efficiencies from the two different standards are differentiated in the figure. Here one may observe some difference between the two standards, with efficiencies deduced from the NBS standard again being the smaller of the two, a difference of $\sim 5 \%$. The more important result is that this check indicates that the present efficiency function is not substantially in error (by, say, 50\%) due to some unknown, indeterminant error in this phase of the experiment. 
ORNL-DWG 90M-15753R

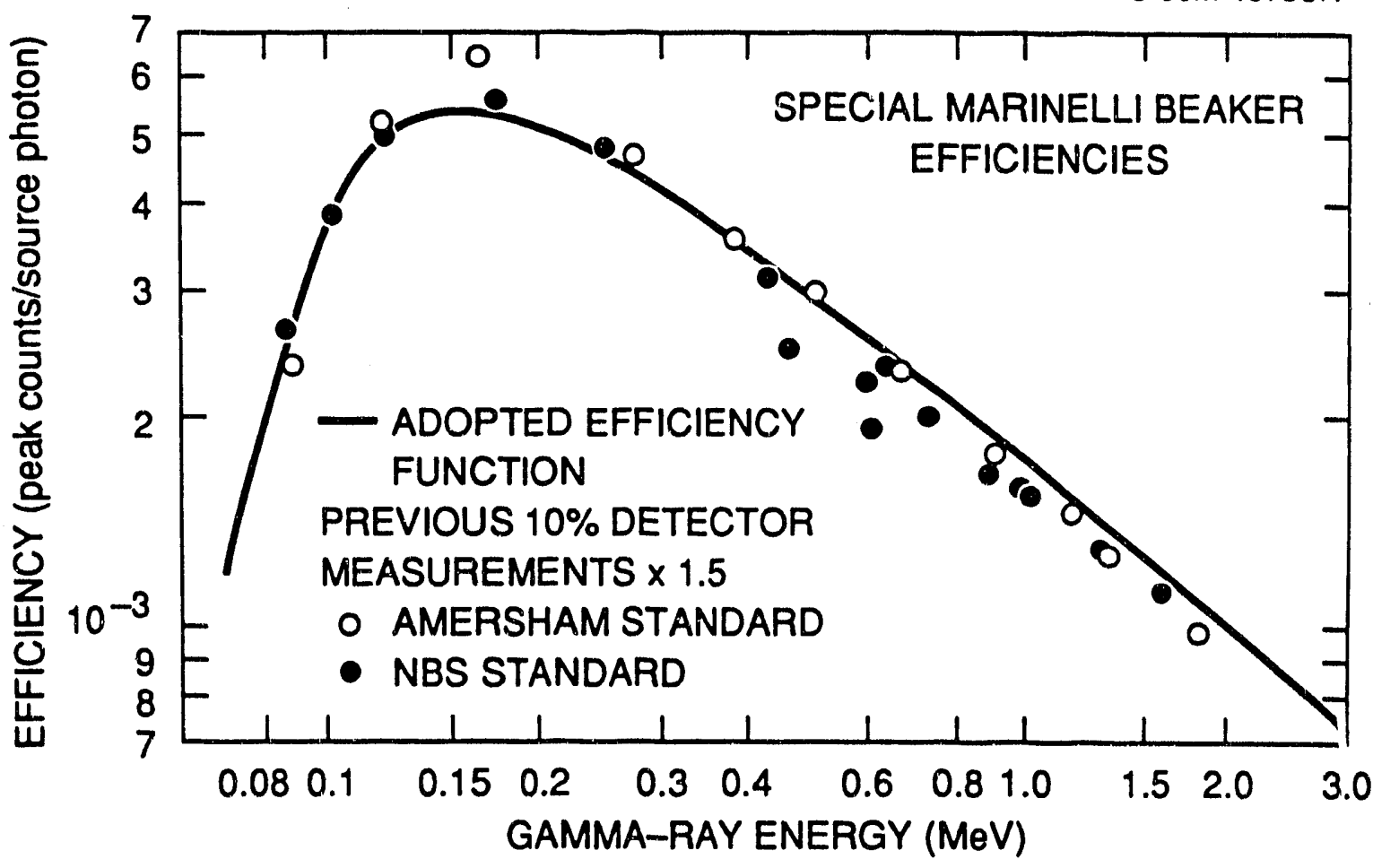

Fig. 3. Comparison of the present adopted efficiency function with adjusted efhciencies of similar detector as given in ref. 9 . 


\section{GAMMA-RAY DATA REDUCTION}

All eleven spectra were first analyzed using the documented analysis computer program TPASS. ${ }^{13}$ The program first deduces a general, and relatively smooth, background/continuum and then finds the peaks superimposed upon the background/continuum. Each peak is analyzed for area and width. An energy calibration is input to the program, and from this calibration the program deduces the mean energy associated with each peak. The efficiency calibration (discussed above) is also input to the program, and from these data the efficienry-corrected yield is determined. Then, following a look-up procedure from a library of radionuclides, the program determines assignments of the found peaks to specific radionuclides. One may input a list of radionuclides for the program to search on. For each such radionuclide found in the library (a separate file on disk ${ }^{14}$ ), the library includes a listing of gamma-ray transitions including the gamma-ray energy and its branching ratio following decay of the chosen radionuclide. The program uses this information to compute the number of atoms of the given radionuclide at the time of the end of the collection period. An uncertainty is also computed. This computation is done for euch gamma-ray ascribed to decay of that specific radionuclide. If the program did not locate a peak of the proper energy in the initial phase then the program is instructed to return to the raw spectral data to determine if it missed a "peak" during the initial phase, and if so to perform a calculation of yield and efficiency-corrected yield. If the program cannot find a "peak" that has a one-standard-deviation statistical uncertainty smaller than $100 \%$ a message will be output indicating this situation. The program will also search the complete library of isotopes to determine if any other comparably formed radionuclide has a strong transition of about the same gamma-ray energy which could interfere with the analysis for the desired radioisotope.

Experience with TPASS indicates that the program adequately analyzes a peak which is "large" with respect to the underlying continuum, and usually satisfactorily analyzes a peak which is "small" with respect to the underlying continuum when it recognizes the existence of such a peak. Occasionally, however, "small" peaks are missed, and so the spectrum must be restudied to ascertain if such peaks are present. This phase of the data reduction utilizes an interactive computer program written (in BASIC) for an IBM-AT personal computer. ${ }^{15}$ This program, called PLOTDATA, displays a portion of the total spectrum (typically 50 channels) on a channel-bychannel basis. The display can be manipulated, for example, by changing limits on either the $x$ or the $y$ axis, or displaying the $y$-axis (i.e., pulse-height data) in either linear or logarithmic scale. The underlying continuum is estimated interactively and when decided upon the program then computes the yield of the peak and its uncertainty. Unlike TPASS, the program PLOTDATA can go no further with the analysis; the rest of the analysis must be done manually.

One radionuclide of particular interest was the radionuclide ${ }^{134} \mathrm{I}$ which has a half life of 52 minutes. A measurable quantity of this isotope $v$ as reported in the earlier (2 June 1989) sampling of the builuing exhaust. ${ }^{1}$ No indication of the presence of this isotope in any of the presently-obtained spectra was recorded in the TPASS analyses; however, the counting periods for the firs four spectra were rather short for a weak transition to have been identified. To enhance somewhat the statistical accuracy the spectral data for the first four gamma ray measurements (runs 


\section{GAMMA-RAY DATA REDUCTION}

27 through 30 in Table 2) were summed. In this manner a "twelfth" experimental spectrum was formed, analyzed using TPASS and then analyzed again using PLOTDATA specifically to ascertain a value for a possible contribution from the radioisotope ${ }^{134} \mathrm{I}$. In addition, the last run of the series (No. 37 in Table 2) was scrutinized carefully for gamma rays indicating the possible presence of two mass 1.33 radioisotopes, I and $\mathrm{Xe}$, also reported ${ }^{1}$ with large unceriainties in the earlier sampling. 


\section{ANALYSIS OF THE REDUCED GAMMA-RAY DATA}

\subsection{FOR ${ }^{41} \mathrm{Ar}$}

The radioisotope ${ }^{41} \mathrm{Ar}$ is created during the reaction of neutron capture by the stable isotope ${ }^{40} \mathrm{Ar}$. This stable isotope mikes up $99.6 \%$ of elemental argon, and elemental argon makes up $0.93 \%$ of ordinary air (see Table 1 ). The "signature" of the presence of ${ }^{41} \mathrm{Ar}$ is detection of the $1294-\mathrm{keV}$ gamma ray following decay of this radioisotope. The peak corresponding to detection of this gamma ray was readily identified in the Sampie No. 1 spectral data; the peak was completely absent in the Sample No. 2 spectral data. See Fig. 4, where the left-hand part of the figure exhit:ts the pertinent portion of the spectrum of run no. 29 for Sample No. 1 and the right-hand part of the figure exhibits the pertinent portion of the spectrum for run no. 33 for Sample No. 2. Clearly, ${ }^{41} \mathrm{Ar}$ was created during the operation of the ORELA; it was absent when the ORELA was turned off.

As mentioned above, the data reduction results in computing the yield of atoms of the contributing radionuclide, in this case ${ }^{41} \mathrm{Ar}$, effective at the end of the collection phase. The results of the 7 measurements (of Table 2) for Sample 1 are exhibited in Fig. 5 where the "measured" values are plotted as a function of time after collection, and the "time after collection" represents the beginning of each specified counting period. (The choice of plotting position along the time axis is not crucial. The time corresponding to the midpoint of the counting period would have been stitable. The important feature is to exhibit the restilts from each separate run.) The uncertainties assigned to each experimental datum are dominated by statistical (counting) uncertainties. The only correlated uncertainty is that of the detector's efficiency for $E_{\gamma}=1294 \mathrm{keV}$. This uncertainty is taken to be $3 \%$; refer to Fig. 2. Thus, these results may be combined ${ }^{16}$ using weighting determined by statistical uncertainties to arrive at a value for the number of ators of ${ }^{41} \mathrm{Ar}$ in Sample No. 1 at the end of the collection period. This va! 2

$$
Y=(1.13 \pm 0.05) \times 10^{6}
$$

is exhibited in Fig. 5 as an open diamond plotted at $\mathrm{T}=0$.

The period of collection, $1.75 \mathrm{hr}$, is almost the same as the half life of ${ }^{41} \mathrm{Ar}$, which is $1.82 \mathrm{hr}$. Thus, to determine the volume density of ${ }^{41} \mathrm{Ar}$ created during ORELA

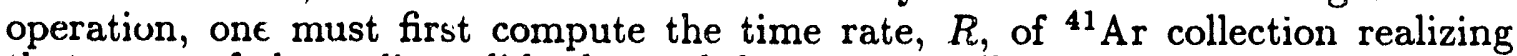
that some of the radionuclide decayed during the collection period. Although as described above the collection rate was not constant, the first assumption is to assume a constant ${ }^{41} \mathrm{Ar}$ collection rate. Then,

$$
Y=R\left(1-e^{-\lambda T}\right) / \lambda
$$

where $\lambda$ is the decay constant for ${ }^{41} \mathrm{Ar}$ and $T$ is the period of collection. The result is,

$$
R=(246 \pm 11) \text { per sec. }
$$




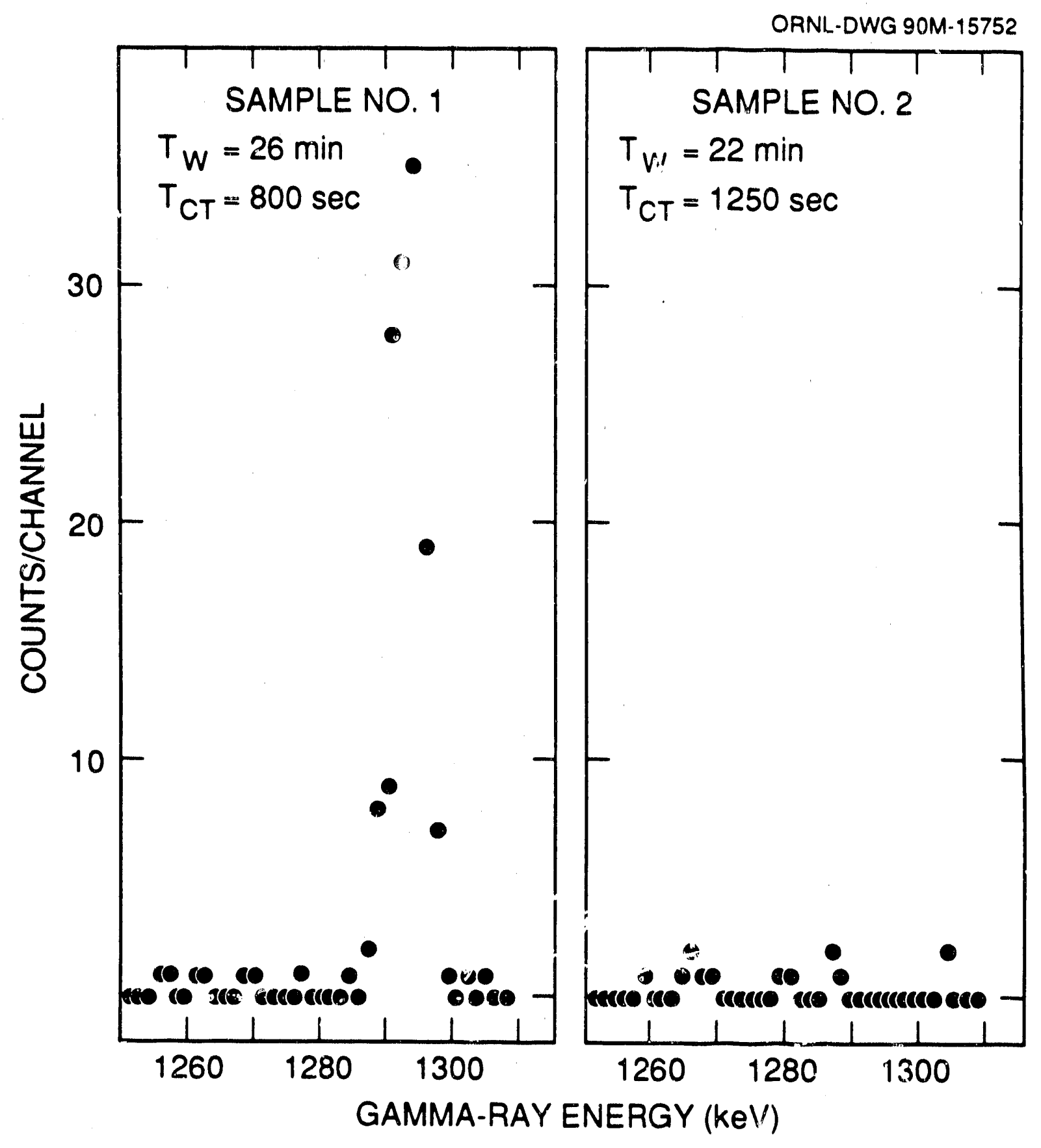

Fig. 4. P. w spectral data corresponding to the gamma-ray energy region includ. ing the $E_{\gamma}=1294$ ke' $/$ gamma ray following decay of " ${ }^{11} \mathrm{Ar}$. $T_{W}$ is the time between the end of the sample collection and the beginning of the counting period, $T_{C T}$. 


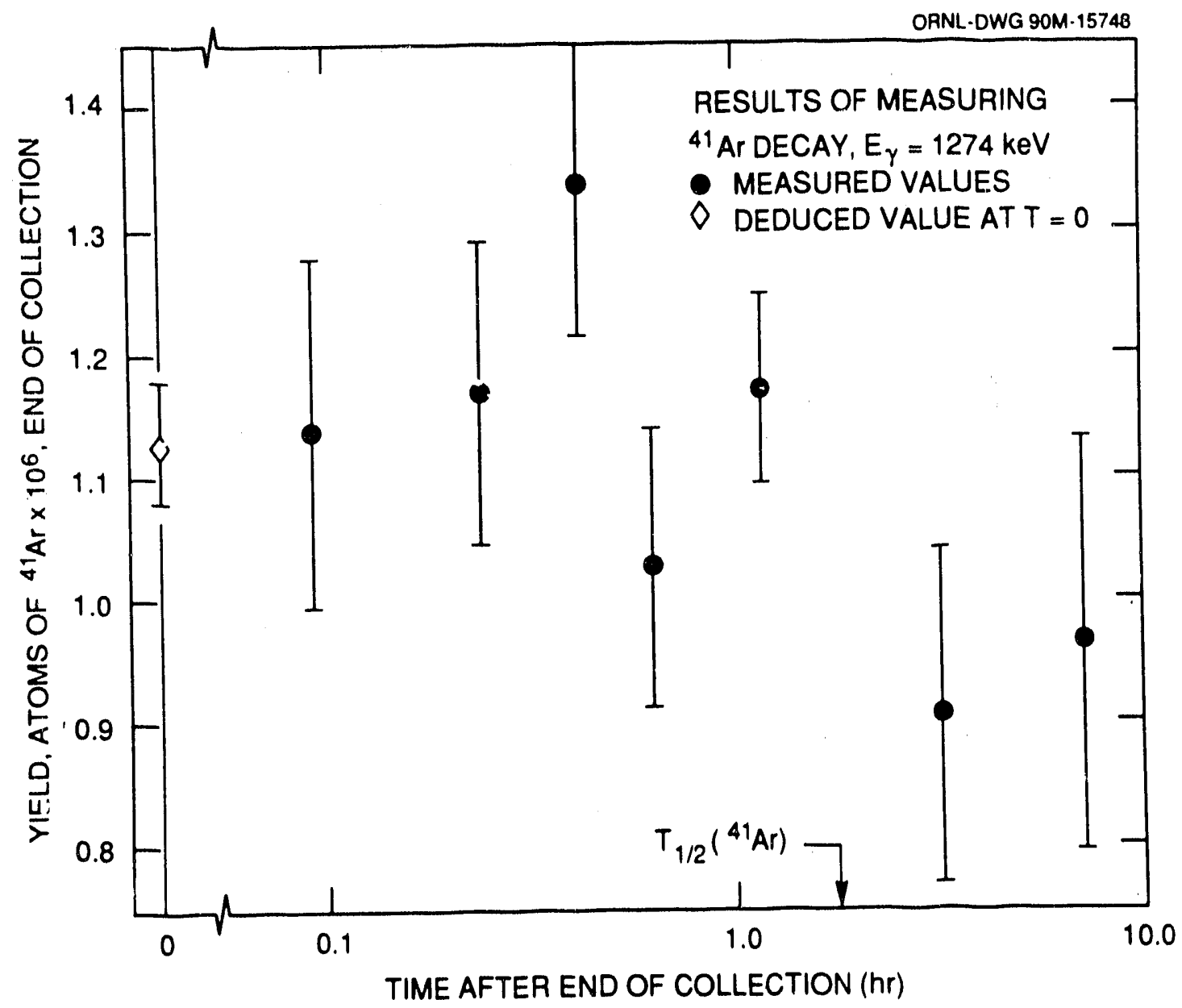

Fig. 5. Results of measuring ${ }^{41}$ Ar decay. The data have been analyzed to give the yield in atoms of ${ }^{41} \mathrm{Ar}$ in the sample at the end of the sample collection period (i.e., at $T=0$ ). 
As discussed above the volume rate of collection assuming a constant rate, $C_{\mathrm{av}}$ of Eq. (1), above, is $3.46 \mathrm{\ell} / \mathrm{min}$, so that the time needed to collect one $\ell$ would be 17.35 sec. So using the assumption of constant rate of collection the number of ${ }^{41} \mathrm{Ar}$ atoms collected per $\ell$ is

$$
N=246 \times 17.35=4271 \text { atoms } / \ell .
$$

Then the decay rate per liter is

$$
N \lambda=0.45 \text { decays } / \mathrm{sec}=12.2 \mathrm{pCi} / \ell .
$$

There are several corrections to be made. The first correction to be considered is the one due to experimental observation that the rate of volume collestion was not constant. This correction was computed by writing a short computer routine to calculate (numerically) the yield of ${ }^{41} \mathrm{Ar}$ for an exponentially declining rate of collection, using the declining decay rate $\alpha$ of Eq. (4). The calculation consisted of computing the number, $Y$, of ${ }^{41} \mathrm{Ar}$ for each second and corrected for decay to the end of the collection period and then summed:

$$
Y_{s}=R_{i} \sum_{S=1}^{6300} e^{-\alpha S} e^{-\lambda(T-S)},
$$

where the first term of the sum represents the (declining) collection rate of ${ }^{41} \mathrm{Ar}$ and the second term gives the correction due to decay of the collected ${ }^{41}$ Ar for the $\mathrm{S}^{\text {th }}$ second. Of course, the initial rate, $R_{i}$, of ${ }^{41} \mathrm{Ar}$ collection in this case is larger than the average rate, $R$, given by $\mathrm{Eq}$. (7). This initial rate is determined by observing that the ratio $R_{\mathrm{i}} / R$ should be approximately the same as the initial volume collection ratio $C_{i} / C_{\mathrm{av}}$. The computed number of ${ }^{41} \mathrm{Ar}$ atoms from this sum is,

$$
Y_{s}=1.10 \times 10^{6}
$$

which, when compared to the value $Y$ of Eq. (5) at $T=0$ on Fig. 5, indicates a $2.7 \%$ increase is needed for the initial rate, $R_{i}$, which, in turn, indicates a $2.7 \%$ increase in the rate, $R$, of Eq. (7).

The second correction to be made is to account for the $(5.5 \pm 0.7) \%$ estimated loss of the collected liquid sample during the transfer to the Marinelli beaker. ${ }^{9}$ Thus, the total corrected ${ }^{41} \mathrm{Ar}$ decay rate from the uncorrected value given in Eq. (9) is

$$
D_{\text {corr }}=12.2 \times 1.027 \times 1.055=13.2 \mathrm{pCi} / \ell,
$$

with a one-standard-deviation uncertainty of $0.7 \mathrm{pCi} / \ell$.

\subsection{FOR ${ }^{134} \mathrm{I}$}

The report of this building's stack measurement of 2 June $1989^{1}$ indicated the detection of the decay of the radionuclide ${ }^{134} \mathrm{I}$. This radioisotope of iodine has a half life of about $53 \mathrm{~min}$, and it is produced in quantity by reactions with neutrons only through the fission process. The report of the identification and quantification of this radionuclide in this building's exhaust was of some concern because the 
mechanism responsible for creating ${ }^{134}$ I during ORELA operation was not apparent. Indeed, one of the goals of the present measurement was io try to determine the source of this isotope.

The decay of ${ }^{134} \mathrm{I}$ results in a number of o'sservable gamma rays, the two principal ones having $E_{\gamma}=847$ and $884 \mathrm{keV}$ with branching ratios of 95.4 and $65.3 \%$, respectively. All of the remaining decay gamma rays have branching ratios less than $20 \%$. As mentioned above, neither of the two principal gamma rays were identified in any of the separate runs 27 through 30 , and so these four runs were summed to give a spectrum in which peaks corresponding to detection of either or both of these two gamma rays might be observed. The relevant portion of this sum spectrum is given in Fig. 6, and the anticipated centroids of potential peaks indicating detection of the two gamma rays are indicated on the drawing. There are no peaks evident to the naked eye in this figure, and an analysis using TPASS failed to detect peaks at these energies.

One may, however, manipulate the raw data to estimate the upper limit of detection for each of these two gamma rays, since it is possible that gamma-ray yields of ${ }^{134} \mathrm{I}$ may have been present in amounts consistent with the earlier measurement ${ }^{1}$ and simply not recorded in the present experiment above the "background" exhibited in the figure. The detection resolution at these energies is such that if a peak had been recorded it would have a full-width-at-half-maximum (FWHM) of about 3 channels. One may assume, therefore, if a "peak" were to have been observed in this spectrum, it would have been spread over 6 to 8 channels (or more if it were a large peak). Hence, the aralysis assumed that the data for eight channels, four on either side of the centroid (which is indicated by the arrow labelled " $847.0 \mathrm{keV}$ " in Fig. 6) contained data due to detection of a gamma ray having $E_{\gamma}=847.0 \mathrm{keV}$, a gamma ray which may or may not have been due to decay of ${ }^{134} \mathrm{I}$. The "background" subtraction was obtained from the sum of the data in the four channels just below the "peak" channels plus the four channels just above the "peak" channels. The difference, then, is the "yield" for the "peak", and the square root of the sum is the uncertainty on this "yield." Numerically,

$$
Y(847.0-\mathrm{keV} \text { "peak" })=37-25=(12 \pm 8) \text { counts } .
$$

A similar analysis was done for a possible "peak" having $E_{\gamma}=884.1 \mathrm{keV}$. The result is,

$$
Y(884.1-\mathrm{keV} \text { "peak" })=22-27=(-5 \pm 7) \text { counts . }
$$

Now applying the detection system's efficiency and the nuclear data for the decay of ${ }^{134} \mathrm{I}$, one obtains for the possible number of ${ }^{134} \mathrm{I}$ atoms at $T=0$, i.e., assuming detection of real ${ }^{134}$ I decay,

$$
\begin{aligned}
& Y\left({ }^{134} \mathrm{I}, 847.0 \mathrm{keV}\right)=(14400 \pm 9570) \text { atoms, and } \\
& Y\left({ }^{134} \mathrm{I}, 884.1 \mathrm{keV}\right)=(-9040 \pm 12700) \text { atoms at } T=0 .
\end{aligned}
$$

The uncertainties are essentially entirely statistical, and these two yields can be combined by weighted least squares ${ }^{16}$ to yield,

$$
Y\left({ }^{134} \mathrm{I}\right)=(5850 \pm 11300) \text { atoms at } T=0 .
$$




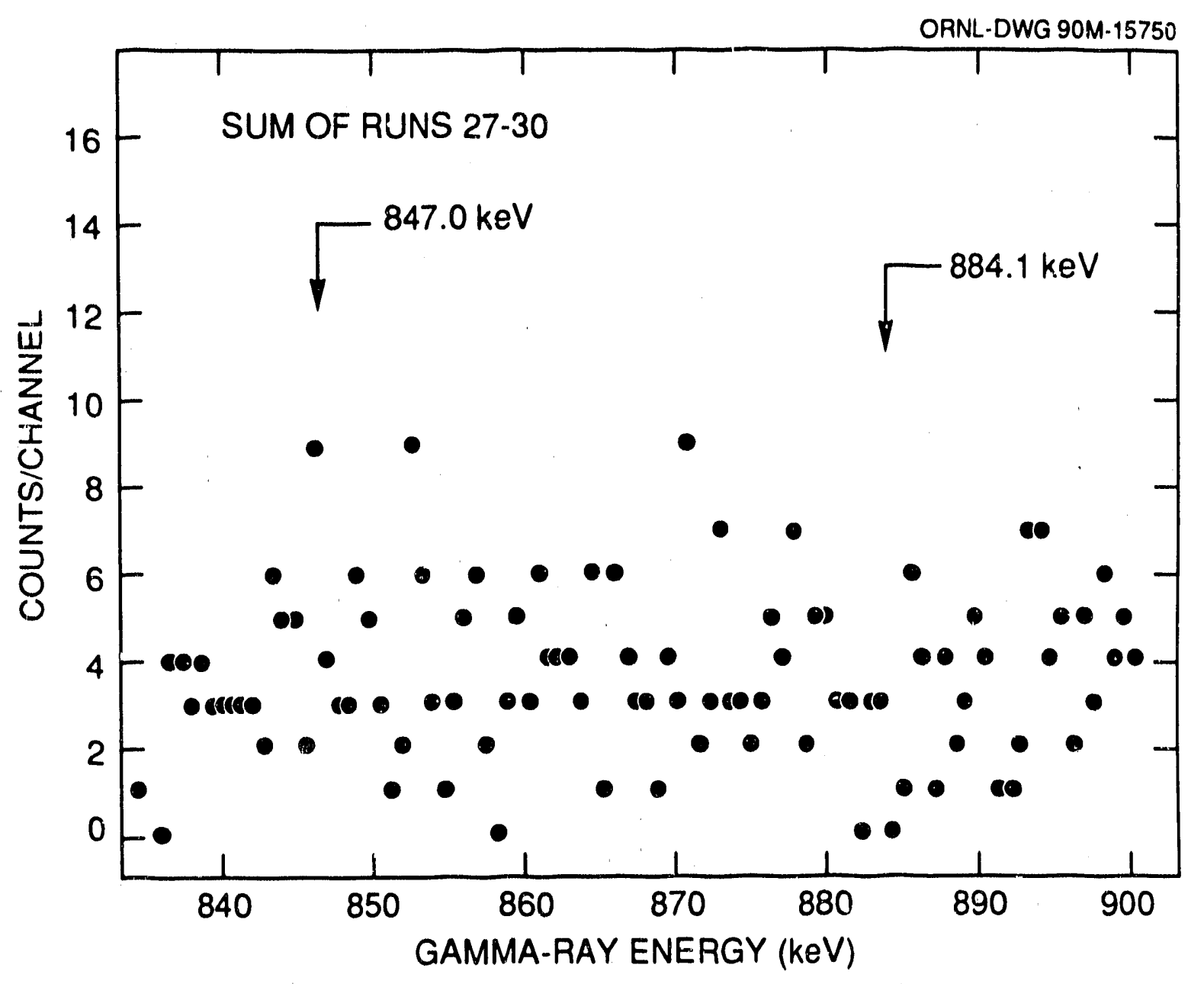

Fig. 6. Relevant portion of the spectrum obtained by summing runs 27 to 30 for the analysis of the presence of ${ }^{134} \mathrm{I}$ in Sample No. 1 . The expected centroids of peaks corresponding to detection of the two principal gamma rays observed in the decay of ${ }^{134} \mathrm{I}$ are exhibited in this figure.

One may note that this weighted-least-squares value is more than twice the simple average value of $\approx 2700$ atoms of ${ }^{134} \mathrm{I}$ at $T=0$. Now the same numerical treatment starting with Eq. (6) is applied to the value of $Y$ in Eq. (15c) only now $\lambda$ is the decay constant for ${ }^{134} \mathrm{I}$. The calculated correction for the non-constant rate of volume collection, Eq. (10), for this shorter-lived isotope is $4.9 \%$. The transfer correction discussed above is the same. The final result for ${ }^{134} \mathrm{I}$ decay observed in the present set of data, is:

$$
D\left({ }^{134} \mathrm{I}\right)=(0.20 \pm 0.38) \mathrm{pCi} / \ell
$$

One could, of course, argue that the completely negative result in the analysis for a "peak" corresponding to detection of the $E_{\gamma}=884.1-\mathrm{keV}$ gamma ray indicates that there was no decay of ${ }^{134}$ I detected, and that whatever "peak" may be ascribed 
to detection of an $E_{\gamma}=847.0-\mathrm{keV}$ gamma ray, that "peak" does not correspond to detection of ${ }^{134} \mathrm{I}$ decay. Such is likely the situation, and all that is stated here is that if one accepts the dejection of an $E_{\gamma}=847-\mathrm{keV}$ gamma ray as an indicatichn of decay of ${ }^{134} \mathrm{I}$ one must also accept the need to detect an $E_{\gamma}=884-\mathrm{keV}$ gamma ray for positive identification of detection of decay of ${ }^{134} \mathrm{I}$; the assumption that ${ }^{134} \mathrm{I}$ existed in the stack-exhaust sample requires an analysis such as given herein, and the result given in Eq. (16) is the numerical best estimate consistent with said assumption. Certainly for practical purposes, this experiment reports a null result.

\subsection{FOR MASS = 133 ISOTOPES}

The report ${ }^{1}$ of this building's earlier stack measurement (of 2 June 1989) also indicated detection if the decay of the radioisotopes ${ }^{133} \mathrm{I}$ and ${ }^{133 m} \mathrm{Xe}$, both isotopes being products of the fission process, but as in the case of ${ }^{134} \mathrm{I}$, neither isotope produced as a consequence of the operation of the ORELA. One would not expect to create a detectable amount of ${ }^{133 m} \mathrm{Xe}$ radioisotope in the ORELA target room in the same manner as the ${ }^{41} \mathrm{Ar}$ was created simply because the amount of Xe in the atmosphere is six orders of magnitude smaller than the amount of $\mathrm{Ar}$ in the atmosphere (see Table 1). And, if, indeed, the capture mechanism were the method of creation, one should also see the other radioisotopes of Xe created by neutron capture by the other stable isotopes of Xe making up natural Xe. For ${ }^{133} \mathrm{I}$, just as for the ${ }^{134} \mathrm{I}$, the mechanism for creation during ORELA operation is simply not apparent.

Because of the earlier report ${ }^{1}$ it was deemed necessary to make the attempt to detect the important gamma rays, viz $E_{\gamma}=233.5 \mathrm{keV}$ from decay of ${ }^{133} \mathrm{Xe}$ and $E_{\gamma}=529.5 \mathrm{keV}$ from decay of ${ }^{133} \mathrm{I}$. The last run, run no. 37 of Table 2 , was initiated 7.25 hours after the end of collection (so that the shorter-lived radioisotopes had completely decayed) and was counted for a little more than 4 hours, the length of time heing dictated, at least partly, by the concern for the anticipated oxygen enrichment of the sample and the late hour in the day of the count. An estimate of the anticipated counting rate was made on the basis of the $0.17 \mathrm{pCi} / \ell$ mean value reported for the 2 June 1989 measurement, ${ }^{1}$ and this estimate was $\sim 15$ counts per hour in the peak corresponding to $E_{\gamma}=529.5 \mathrm{keV}$. This counting rate, it should be acknowledged, was about one-third the initially observed total counting rate for 8 channels of pulse-height-analyzer data having a centroid corresponding to the desired gamma ray. Even so, in four hours, one might anticipate a sufficiently welldefined peak in the spectral data to, at least, make an identification. One could not be so sanguine as to expect in 4 hours to observe much of a "peak" for the $E_{\gamma}=233.5 \mathrm{keV}$ decay of ${ }^{133 \mathrm{~m}} \mathrm{Xe}$. The branching for this gamma-ray decay is only $9.9 \%$ compared to $87.3 \%$ for the $E_{\gamma}=529.5-\mathrm{keV}$ decay of ${ }^{133} \mathrm{I}$; and, in addition, the overall counting rate in this lower-energy region was observed to be about 4 times larger than for the region corresponding to $\approx 530 \mathrm{keV}$, negating somewhat the advantage in detection-system efficiency for the lower-energy gamma ray.

The relevant regions of the spectrum from run no. 37 are shown in Fig. 7, and the expected centroids for possible peaks corresponding to detection of the two gamma rays of interest are indicated by the arrows in this figure. Again, there are no peaks evident to the naked eye, nor were any discerned during the TPASS analysis. Manipulating the raw spectral data using the same prescription as described above 

for the two ${ }^{134} \mathrm{I}$ gamma rays resulted in the determining possible "peak" yields as
follows:

$$
\begin{aligned}
Y\left({ }^{133 m} \mathrm{Xe}, 233.5 \mathrm{keV}\right) & =(-11 \pm 59) \text { counts, and } \\
Y\left({ }^{133} \mathrm{I}, 529.5 \mathrm{keV}\right) & =(0 \pm 20) \text { counts. }
\end{aligned}
$$

Applying the formalism already described above, including corrections (the correction for the non-constant gas volume collection being essentially zero for these longer-lived radioisotopes) resulted in disintegration/volume rates as follows:

$$
\begin{aligned}
D\left({ }^{133 m} \mathrm{Xe}\right) & =(-0.12 \pm 0.59) \mathrm{pCi} / \ell, \text { and } \\
D\left({ }^{133} \mathrm{I}\right) & =(0.00 \pm 0.53) \mathrm{pCi} / \ell .
\end{aligned}
$$

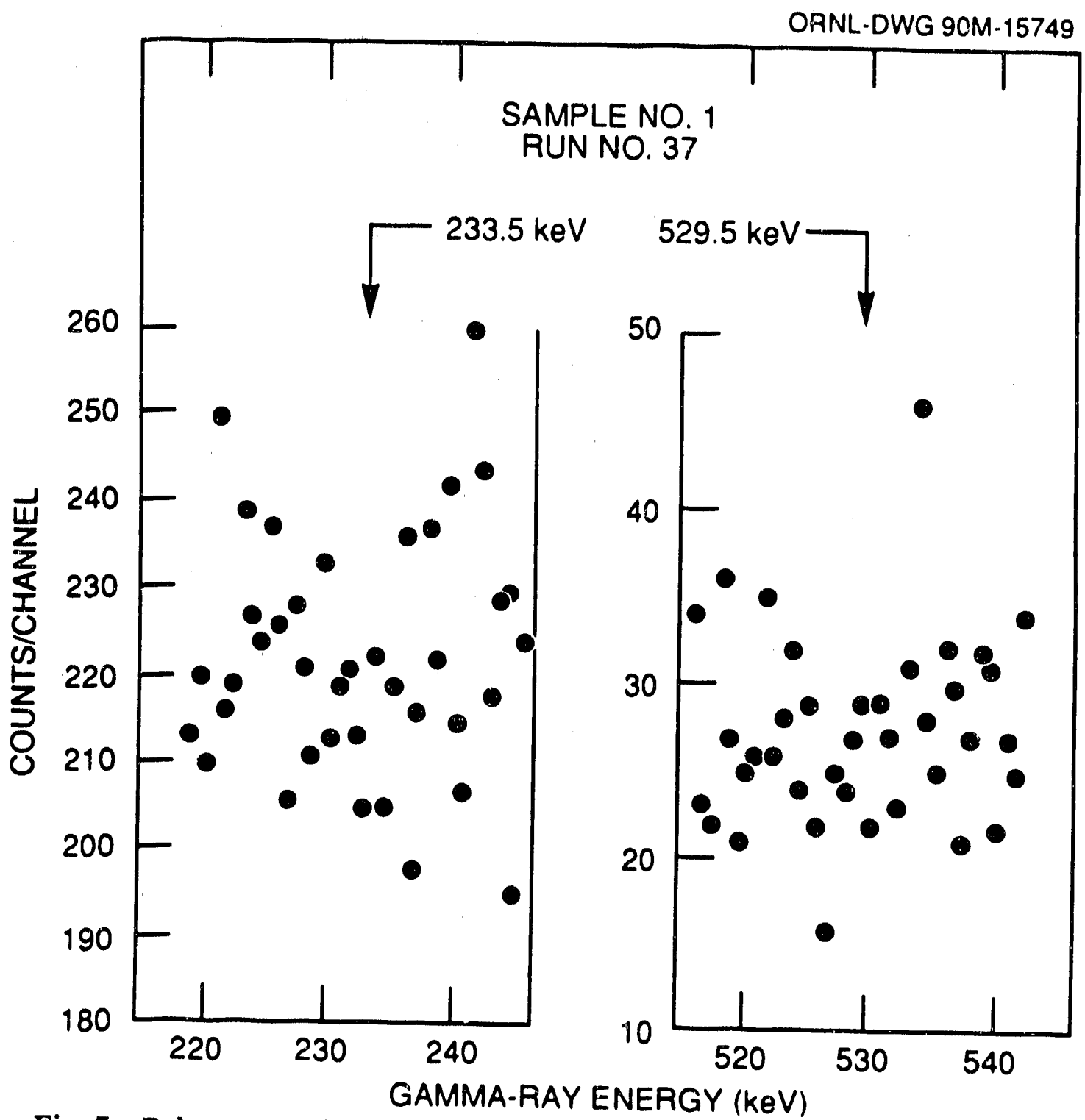

Fig. 7. Relevant portions of the final spectrum for the analysis of the presence of ${ }^{133 m} \mathrm{Xe}$ and ${ }^{133} \mathrm{I}$. The expected centroids of two peaks corresponding to detection of an

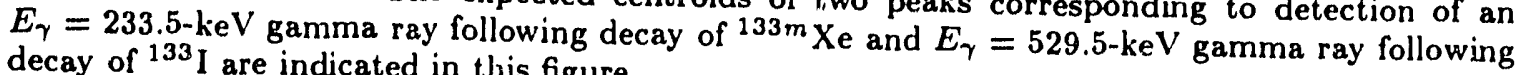




\subsection{FOR ${ }^{222} \mathrm{Rn}$}

All isotopes of the element radon are radioactive. The one of interest is the $T_{1 / 2}=3.2$-day isotope ${ }^{222} \mathrm{Rn}$, which is a daughter of the $T_{1 / 2}=1600$-yr radium isotope ${ }^{226} \mathrm{Ra}$. In an undisturbed environment, this isotope of radon is found in the atmosphere (see Table 1) to varying amounts, with larger concentrations usually indicative of nearby uranium-bearing ore. There is no known mechanism by which ${ }^{222} \mathrm{Rn}$ can be created by ORELA operation. Should an excessive amount be detected in the building's stack exhaust, the source would most likely be ${ }^{226} \mathrm{Ra}$ in a quantity large enough to be of concern.

Analysis for ${ }^{222} \mathrm{Rn}$ utilized the $E_{\gamma}=351.9-\mathrm{keV}$ gamma ray following decay of the daughter radioisotope ${ }^{214} \mathrm{~Pb}$. As mentioned above, this decay is seen also as part of the background emanating from the room environment (along with emanations from ${ }^{40} \mathrm{~K}$ and ${ }^{228} \mathrm{Th}$ ). A measure of the room background contribution was obtained during run no. 36. In Fig. 8 are shown relevent portions of 3 spectra, viz., runs 34 , 35, and 36. Run 34 has data for Sample No. 1, run 35 for Sample No. 2. Referring back to Table 2, run 34 was counted for $50 \mathrm{~min}$ while runs 35 and 36 were for 60 min, and one may observe that the peaks in the spectra for runs 34 and 35 are approximately the same, and they have about twice the yield of the peak in run 36 shown for the background.

Since what was expected was observed, namely the equality of yield in the results for Sample No. 1 with that of Sample No. 2, it was decided to use the data for $E_{\gamma}=$ $351.9 \mathrm{keV}$ in run no. 37 because the siatistical accurary is better. Indeed, as may be deduced from perusal of the right-hand part of Fig. 8, the major uncertainty in the overall analysis to obtain the foreground yield is due to the statistical uncertainty assigned to the yield analysis of the background peak for $E_{\gamma}=351.9 \mathrm{keV}$. From these data one may deduce a value for the ${ }^{214} \mathrm{~Pb}$ disintegration rate. These results are as follows:

$$
\begin{aligned}
& C(\text { Sample No. } 1, \text { run } 37)=(17.2 \pm 1.0) \mathrm{disin} t / \mathrm{sec} \\
& C(\text { Sample No. } 3, \text { run } 36)=(9.6 \pm 2.8) \mathrm{disint} / \mathrm{sec}
\end{aligned}
$$

The difference, ascribed to the detection of ${ }^{222} \mathrm{Rn}$ in the stack exhaust, before any corrections are applied, is:

$$
R\left({ }^{214} \mathrm{~Pb} \text { in stack }\right)=(7.6 \pm 3.0) \mathrm{disint} / \mathrm{sec} .
$$

This disintegration rate, of Eq. (19c), is the rate for the ${ }^{214} \mathrm{~Pb}$ in the sample at about the end of run 36 and the beginning of run 37 , or about $26000 \mathrm{sec}$ after the end of the collection of Sample No. 1. The half life of ${ }^{214} \mathrm{~Pb}$ is $26.8 \mathrm{~min}$ which is a factor of $\sim 16$ smaller than the time from the end of the collection to the start of the counting of run 37 . Thus, one may take the decay of ${ }^{214} \mathrm{~Pb}$ to be in equilibrium with the decay of its parent (actually grandparent) radioisotope ${ }^{222} \mathrm{Rn}$, and so the disintegration rate of Eq. (19c) is that of ${ }^{222} \mathrm{Rn}$ about $26000 \mathrm{sec}$ after the end of the collection. Whatever ${ }^{214} \mathrm{~Pb}$ was in the sample at the end of the collection had essentially no effect. Furthermore, one may treat the collection in either Sample 


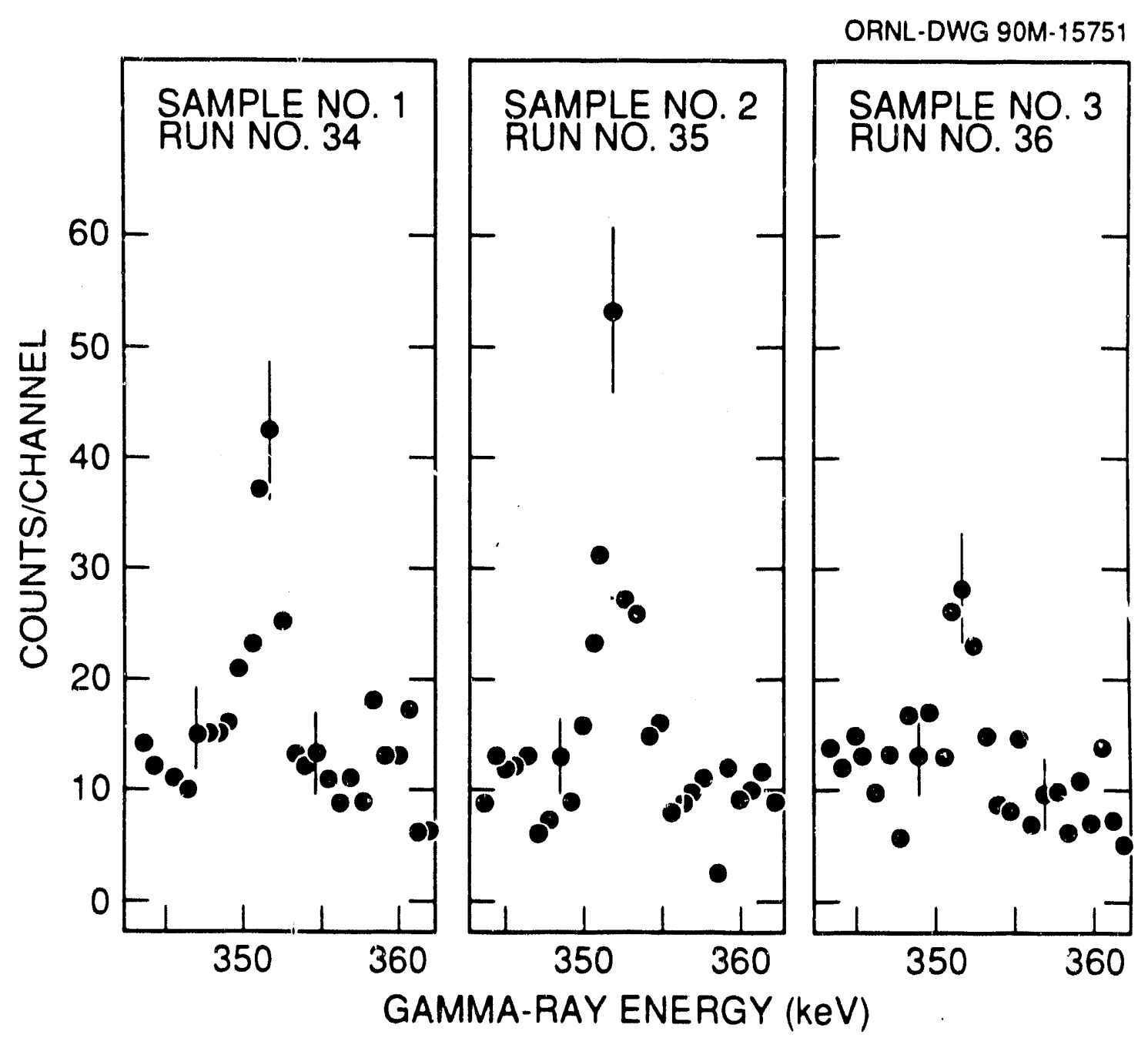

Fig. 8. Relevant portions of three spectra for the analysis of the presence of ${ }^{222} \mathrm{Rn}$ in the sample. 
No. 1 or Sample No. 2 of ${ }^{222} \mathrm{Rn}$ as constant. All that must be done is to apply the correction for the half life of ${ }^{222} \mathrm{Rn}$ to convert the value at $T=26000 \mathrm{sec}$ to a value at $T=0 \mathrm{sec}$, and then apply the above-described correction for loss-of-sample during transfer. The final result is:

$$
D\left({ }^{222} \mathrm{Rn}\right)=(0.62 \pm 0.25) \mathrm{pCi} / \ell .
$$

\subsection{FOR ${ }^{13} \mathrm{~N}$ AND ${ }^{11} \mathrm{C}$}

The decay of the $T_{1 / 2}=10-\mathrm{min}$ radioisotope ${ }^{13} \mathrm{~N}$ is entirely by $\beta^{+}$emission. This mode of radioactivity is detected because the $\beta^{+}$particle annihilates with an ordinary electron producing "annihilation radiation" having a gamma-ray energy of $511.0 \mathrm{keV}$, and the $511.0-\mathrm{keV}$ radiation is observed as a slightly broadened peak in the gamma-ray spectra obtained in thest measurements. Annihilation radiation is observed in room background; it is unresolved from another room background radiation due to decay of ${ }^{228} \mathrm{Th}$ (also known as $\mathrm{ThC"}$ ") at $E_{\gamma}=510.8 \mathrm{keV}$. A peak ascribed to detection of annihilation radiation was observed in every one of the measured spectra; the yield, however, was largest in run no. 27 as expected if the short-lived nitrogen radioisotope were to be detected.

The first step in the data reduction was to determine the count rate (in counts/sec) for each run. The results are shown in Fig. 9, where the data for Samples 1 and 2 are plotted with respect to the cooling time, i.e., the time following the end of the liquid sample collection. The datum for Sample no. 3, indicated by the solid triangle, gives an indication of the room background. It is plotted, for convenience, at a cooling time between the final two measurements of Sample no. 1 , or approximately when the measurement was made in real time. This background value is the smallest of all of the measurements; however, it is not significantly smaller than the three measurements of Sample no. 2 (open triangles) nor the final two measurements of Sample no. 1 (open circles). So, for the purpose of defining a background subtraction for the first five measurements of Sample no. 1, a weighted least-squares ${ }^{16}$ average of these six measurements (i.e., the background run, the three runs for Sample no. 2, and the two final runs for Sample no. 1) was obtained. The value obtained and used for the background subtraction was:

$$
B=(20.6 \pm 1.2) \times 10^{-3} \text { counts } / \mathrm{sec},
$$

a value which is within the measured statistical accuracy of all six data points used in its determination.

The next step was to subtract the background using the rate, $B$, of Eq. (21) from the peak yields obtained for runs 27 to 31 for Sample no. 1. Then came the most difficult part of the data analysis. It was evident that the backgroundcorrected yields for $E_{\gamma}=511 \mathrm{keV}$ were not due to observation of the decay of only one radionuclide, namely ${ }^{13} \mathrm{~N}$. The data given in Table 3 elucidate this point. 


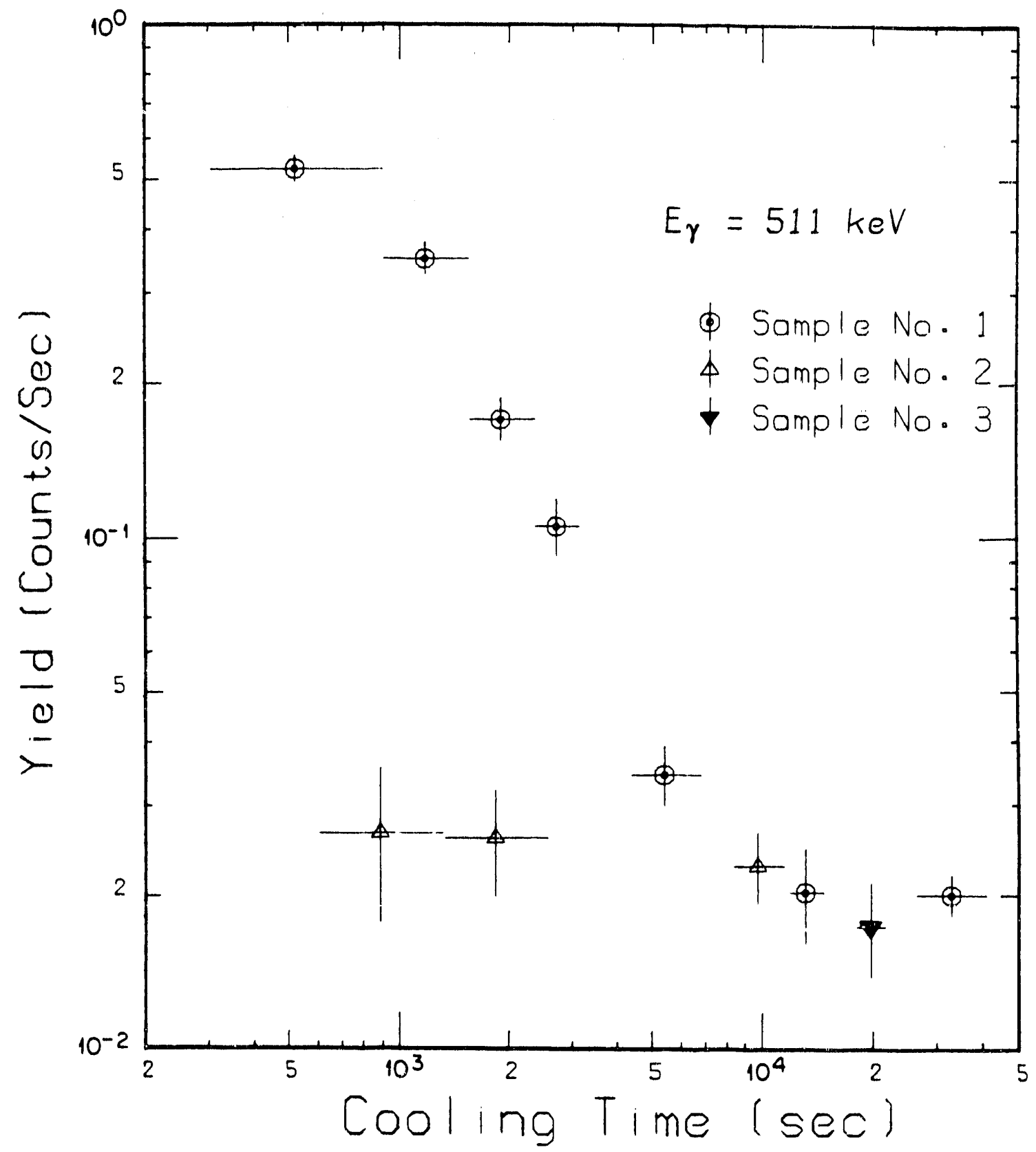

Fig. 9. Counting rate for the peak corresponding to detection of annihilation radiation as a function of the time following the end of sample collection. The horizontal bars indicate the counting period and the vertical bars indicate the statistical uncertainty assigned to the plotted datum. 
Table 3. Comparison of Background-corrected Experimental Yields for $E_{\gamma}=511 \mathrm{keV}$ with Computed Yields for Decay of ${ }^{13} \mathrm{~N},{ }^{15} \mathrm{O}$, and ${ }^{11} \mathrm{C}$. The Computed Yields are Based on an Initial $(T=0)$ Concentration of 500 Atoms of Radionuclide.

\begin{tabular}{cccccc}
\hline Run $^{a}$ & $\mathrm{~T}^{b}$ & Experimental & \multicolumn{3}{c}{ Computed Yield } \\
\cline { 4 - 6 } No. & $(\mathrm{sec})$ & Yield $^{c}$ & ${ }^{13} \mathrm{~N}^{d}$ & ${ }^{15} \mathrm{O}^{d}$ & ${ }^{11} \mathrm{C}^{e}$ \\
\hline 27 & 300 & $301.9+21.1$ & 354 & 48 & 198 \\
28 & 900 & $205.5+17.5$ & 181 & 2 & 145 \\
29 & 1540 & $119.0+14.1$ & 101 & & 123 \\
30 & 2340 & $67.9+11.1$ & 40 & & 78 \\
31 & 4320 & $34.5+12.3$ & 6.3 & & 53 \\
\hline
\end{tabular}

a See Table 2.

${ }^{b} \mathrm{~T}=0$ at the end of sample collection.

${ }^{c}$ In peak counts not corrected for detector efficiency.

${ }^{d}$ Two $E_{\gamma}=511-\mathrm{keV}$ photons/decay.

e $1.62 \Sigma_{\gamma}=511-\mathrm{keV}$ photons/decay.

It is apparent from a quick perusal of the results given in the third and fourth columns of Table 3 that the experimental yield for $E_{\gamma}=511 \mathrm{keV}$ decreases more slowly than would be expected from the decay of the $T_{1 / 2}=598$-sec radionuclide ${ }^{13} \mathrm{~N}$.

Although the evidence is more inferential than overt, the data do not support identification of detection of decay of the $T_{1 / 2}=122-\mathrm{sec}$ radionuclide ${ }^{15} \mathrm{O}$. If the initial amount of ${ }^{15} \mathrm{O}$ were comparable to the initial amount of ${ }^{13} \mathrm{~N}$, then the $E_{\gamma}=$ $511-\mathrm{keV}$ peak yield for run no. 27 would exhibit an enhancement relative to the $E_{\gamma}=511-\mathrm{keV}$ peak yield for run no. 28 , whereas the measured relationship seems to be reversed. One might inquire if the initial amount of ${ }^{15} \mathrm{O}$ relative to the initial amount of ${ }^{13} \mathrm{~N}$ might be estinnated.

To make such an estimate, one may consider the two reactions producing these two radionuclides. One reaction is the photoneutron effect:

$$
\begin{aligned}
& { }^{14} \mathrm{~N}+\gamma \rightarrow n+{ }^{13} \mathrm{~N}, \text { and } \\
& { }^{16} \mathrm{O}+\gamma \rightarrow n+{ }^{15} \mathrm{O} .
\end{aligned}
$$

The other reaction is the $(n, 2 n)$ reaction:

$$
\begin{aligned}
& { }^{14} \mathrm{~N}+n \rightarrow 2 n+{ }^{13} \mathrm{~N}, \text { and } \\
& { }^{16} \mathrm{O}+n \rightarrow 2 n+{ }^{15} \mathrm{O} .
\end{aligned}
$$

One may assume that possible reactions with other stable isotopes of $\mathrm{N}$ and $\mathrm{O}$ would contribute very little to the production of either radionuclide because the ot'.ler stable isotopes make up only a very small fractions of elemental $\mathrm{N}$ and $\mathrm{O}$. 
The first reactions, Eqs. (20a) and (20b), would be due to the higher energy component of the bremsstrahlung. One may estimats relative yields of the radioactive reaction products by observing that the photonuclear uross section, on the average, behaves like ${ }^{17}$

$$
\sigma(n, \gamma) \sim\left(N_{n}\right)^{1.6}
$$

where $N_{n}$ is the sample neutron number. Thus, the ratio

$$
R=\frac{N\left({ }^{15} O\right)}{N\left({ }^{13} N\right)}
$$

at the end of the collection due to bremmstrahlung reactions becomes,

$$
R_{\gamma, n}=\frac{N\left({ }^{16} O\right) \sigma\left({ }^{16} O\right)}{\lambda\left({ }^{15} O\right)} \times \frac{\lambda\left({ }^{13} N\right)}{N\left({ }^{14} N\right) \sigma\left({ }^{14} N\right)}
$$

or,

$$
R_{\gamma, n}=\left(\frac{20.05}{78.09}\right) \times\left(\frac{8}{7}\right)^{1.6} \times\left(\frac{122}{598}\right)=0.068
$$

The first term in parentheses in Eq. (23c) is the ratio of ${ }^{10} \mathrm{O} /{ }^{14} \mathrm{~N}$ from Table 1 , the second term is the ratio of cross sections from Eq. (22), and the third term is the ratio of inverse decay constants.

However, the bremsstrahlung beam due to electron interactions with either the Ta target or the Ta converter is confined to a small cone centered about a vector defining the incident electron bearn direction. The total volume in the target room in which bremsstrahlung-induced reactions could take place is $<1 \mathrm{cu}$. $\mathrm{ft}$. On the other hand, high-energy neutrons produced in the $\mathrm{Ta}$ or Be targets emanate into $4 \pi$ sr, and so the neutron-induced $(n, 2 n)$ reactions of Eqs. (21a) and (21b) can occur throughout the $1700 \mathrm{cu}$. $\mathrm{ft}$. volume of the target room. Determining the ratio, $R$ of Eq. (23a) at the end of the sample stack collection due to $(n, 2 n)$ reactions is not quite so straightforward as was done for Eq. (23b) because the ratio of the cross sections,

$$
R_{\sigma}=\frac{\sigma_{n, 2 n}\left({ }^{16} O\right)}{\sigma_{n, 2 n}\left({ }^{14} N\right)}
$$

is a function of neutron energy and would have to be known for all neutron energies. Some measurements exist. For example, Brill' et al. ${ }^{18}$ reported measurements of both cross sections in Eq. (24) at various incident neutron energies between about threshold ( $\sim 12 \mathrm{MeV}$ for ${ }^{14} \mathrm{~N}$ and $\sim 17 \mathrm{MeV}$ for ${ }^{10} \mathrm{O}$ ) and $40 \mathrm{MieV}$. These measurements indicate that both cross sections peak at $\sim 18 \mathrm{mb}$ for $E_{n}$ between 25 and 30 $\mathrm{MeV}$, and that $R_{\sigma}$ of Eq. (24) is $\approx 1$ for $E_{n}>25 \mathrm{MeV}$. For $E_{n}<25 \mathrm{MeV}, R_{\sigma}$ is $<1$. On the basis that these measurements yield valid representations of the two $(n, 2 n)$ excitation functions, then the ratio equivalent, $R_{n, 2 n}$, to $R_{\gamma, n}$ of Eq. (23c), has a reasonably deduced upper limit:

$$
R_{n, 2 n} \leq 0.06
$$

The total ratio, $R$ of Eq. (23a), is some linear combination of $R_{\gamma, n}$ and $R_{n, 2 n}$. Thus the amount of ${ }^{15} \mathrm{O}$ at the end of the sample collection is expected to be small 
with respect to the amourt ${ }^{13} \mathrm{~N}$, too small to have been clearly identified in the present measurements.

One may ask, "Can the experimental data be accounted for by assuming they are due to detection of decay of only two radionuclides, namely the $T_{1 / 2}=10 \mathrm{~min}$ isotope ${ }^{13} \mathrm{~N}$ and the $T_{1 / 2}=20.3-\mathrm{min}$ isotope ${ }^{11} \mathrm{C}$ ?" In fact, one can show that the data can be so accounted for. The arithmetic (i.e., matrix) operations ${ }^{19}$ to arrive at initial $(T=0)$ concentrations of the two radionuclides are as follows: consider a 5-element column matrix representing the experimental yields,

$$
\mathbf{D}=\left(\begin{array}{c}
301.9 / \epsilon \\
205.5 / \epsilon \\
119.0 / \epsilon \\
67.9 / \epsilon \\
34 . b / \epsilon
\end{array}\right)
$$

The detector efficiency for $E_{\gamma}=511 \mathrm{keV}$ is represented by $\epsilon$. Next consider a square $5 \times 5$ matrix representing the variances and covariances of these data,

$$
\mathrm{V}_{\mathbf{D}}=\left(\begin{array}{ccccc}
445.2 / \epsilon^{2} & \cdot & \cdot & \cdot & \cdot \\
\cdot & 306.3 / \epsilon^{2} & \cdot & \cdot & \cdot \\
\cdot & \cdot & 198.8 / \epsilon^{2} & \cdot & \cdot \\
\cdot & \cdot & \cdot & 123.2 / \epsilon^{2} & \cdot \\
\cdot & \cdot & \cdot & \cdot & 151.3 / \epsilon^{2}
\end{array}\right)
$$

The diagonal terms will be recognized as the squares of the uncertainties assigned to the experimental data as given in Table 3 . The off-diagonal terms (here left blank) represent correlations among the five data. In the present experiment there is a small correlation among the data due to the uncertainty in the detector efficiency, $\Delta \epsilon$. Since $\epsilon$ is the only correlation, $\Delta \epsilon$ could be factored out and then reinstated in the final results. What was done in the present analysis was to assume that $\Delta \epsilon$ was small compared to the statistical uncertainties; on the basis of this assumption all of the off-diagoral terms of $V_{D}$ were set equal to zero.

Finally, consider a $2 \times 5$ column array representing the "theoretical" values for ${ }^{13} \mathrm{~N}$ and ${ }^{11} \mathrm{C}$ given in the fourth and sixth columns of Table 3 , only now for an initial concentration of 1 atom:

$$
\mathbf{G}=\left(\begin{array}{cc}
0.708 & 0.395 \\
0.362 & 0.290 \\
0.203 & 0.247 \\
0.080 & 0.156 \\
0.013 & 0.105
\end{array}\right)
$$

Let $I$ be a two-row vector representing least-squares estimates of the initial concentrations of ${ }^{13} \mathrm{~N}$ and ${ }^{i 1} \mathrm{C}$; then, in matrix representation,

$$
I=\left(G^{+} V_{D}^{-1} G\right)^{-1} G^{+} V_{D}^{-1} D
$$




\section{ANALYSIS OF THE REDUCED GAMMA-RAY DATA}

where $G^{+}$is the adjoint matrix to $\mathbf{G}$, i.e, a 5 -column by 2 -row matrix. The $2 \times 2$ covariance matrix of $I$ is given by,

$$
\mathbf{V}_{\mathbf{I}}=\left(\mathbf{G}^{+} \mathbf{V}_{\mathbf{D}}{ }^{-1} \mathbf{G}\right)^{-1}
$$

This formalism is exact in the least-squares sense for this problem.

The results of this calculation are as follows:

$$
\begin{aligned}
N\left({ }^{13} N\right) & =(9.40 \pm 1.86) \times 10^{4} \text { atoms at } T=0, \text { and } \\
N\left({ }^{11} C^{C}\right) & =(9.92 \pm 2.28) \times 10^{4} \text { atoms at } T=0 .
\end{aligned}
$$

Ons: may now compare the predicted yield, $P$, as a function of time after the end of the collection period of the $E_{\gamma}=511 \mathrm{keV}$ gamma ray with the experimental yield, $Y_{\exp }$. 1 goodness-of-fit parameter, $\chi^{2}$, is computed for each experimental and corresponding theoretical datum as,

$$
\chi_{i}^{2}=\left[\left(Y_{\exp }-P\right) / \Delta Y_{\exp }\right]^{2} .
$$

For all five experimental-theoretical comparisons, a reduced $\chi^{2}$ is computed as follows,

$$
\chi_{\text {reduced }}^{2}=\frac{1}{N-\nu} \sum_{i=1}^{5} \chi_{i}^{2}
$$

where $N=5$ is the number of comparisons and $\nu=2$ is the number of degrees of freedom. Generally, a reduced $\chi^{2} \leq 1$ indicates that the assumed theoretical interpretation is adequate. ${ }^{20}$ The results of the present analysis are given in Table 4.

Table 4. Analysis of yields for $E_{\gamma}=511 \mathrm{keV}$ as a sum of computed yields for the decay of ${ }^{13} \mathrm{~N}$ and ${ }^{11} \mathrm{C}$.

\begin{tabular}{lcrcrc}
\hline Run & Experimental & \multicolumn{3}{c}{ Computed Yield } & $\chi_{i}^{2 a}$ \\
\cline { 3 - 5 } No & Yield $^{b}$ & \multicolumn{1}{c}{$\mathrm{N}^{c}$} & $\mathrm{C}^{d}$ & Sum $^{e}$ & \\
\hline 27 & $102700 \pm 7200$ & 66600 & 39200 & 105800 & 0.18 \\
28 & $69900 \pm 5950$ & 34000 & 28800 & 62800 & 1.43 \\
29 & $40500 \pm 4800$ & 19100 & 24500 & 43600 & 0.42 \\
30 & $23100 \pm 3800$ & 7500 & 15500 & 23000 & 0.00 \\
31 & $11700 \pm 4200$ & 1200 & 10400 & 11600 & 0.00 \\
\hline
\end{tabular}

${ }^{a}$ See Eq. (31).

${ }^{b} Y_{\operatorname{sxp}}$ of Eq. (31), data of Table 3 corrected for efficiency.

${ }^{c}$ Basis $9.40 \times 10^{4}$ atoms at $\mathrm{T}=0, \mathrm{Eq}$. (30a).

${ }^{d}$ Basis $9.92 \times 10^{4}$ atoms at $\mathrm{T}=0$, Eq. (30b).

$e$ Sum $\equiv P$ of Eq. (31). 
The reduced $\chi^{2}$ of Eq. (32) is,

$$
\chi_{\text {reduced }}^{2}=0.68,
$$

and so the background-subtracted yields of the first five spectra can be accounted for as detection of the sum of the radiations following the decays of only ${ }^{13} \mathrm{~N}$ and ${ }^{11} \mathrm{C}$.

Having now deduced the $T=0$ values of the numbers of atoms of the two radionuclides, ${ }^{13} \mathrm{~N}$ and ${ }^{11} \mathrm{C}$, one may now do the same mathematical analyses as done above for ${ }^{41} \mathrm{Ar}$, Eqs. (6) to (12), only substituting the decay constants for ${ }^{13} \mathrm{~N}$ and ${ }^{11} \mathrm{C}$, respectively. The uncorrected radionuclide concentrations are calculated to be,

$$
\begin{aligned}
& Y_{s}\left({ }^{13} \mathrm{~N}\right)=59 \mathrm{pCi} / \ell, \text { and } \\
& Y_{s}\left({ }^{11} \mathrm{C}\right)=15.5 \mathrm{pCi} / \ell
\end{aligned}
$$

The correction for non-constant volume gas collection is $17.5 \%$ for ${ }^{13} \mathrm{~N}$ and $11.5 \%$ for ${ }^{11} \mathrm{C}$. The transfer correction is the same as for the other analyses. So, finally, one obtains the corrected decay rates per unit volume for these two radionuclides:

$$
\begin{aligned}
& D\left({ }^{13} \mathrm{~N}\right)=(73 \pm 14) \mathrm{pCi} / \ell, \text { and } \\
& D\left({ }^{11} \mathrm{C}\right)=(18.2 \pm 4.2) \mathrm{pCi} / \ell
\end{aligned}
$$

\subsection{OTHER RADIONUCLIDES}

The only other peaks observed in any of the experimental spectra were assigned to known gamma-ray transitions following decay of previously observed background. These included, for example $E_{\gamma}=1460 \mathrm{keV}$ due to decay of ${ }^{40} \mathrm{~K}$ and $E_{\gamma}=2614$ $\mathrm{keV}$ due to decay of a daughter of ${ }^{228} \mathrm{Th}$. All such background peaks were small, and, except as discussed above, did not interfere with analyses of peaks of interest. 


\section{SUMMARY AND CONCLUSIONS}

Samples of the gaseous components of the Building 6010 exhaust stack were obtained for two conditions, (a) the ORELA in a normal operating mode, and (b) the ORELA shut off. The decay of one radionuclide, ${ }^{222} \mathrm{Rn}$, was observed equally in both samples. The characteristic radiations due to decay of three radionuclides, namely ${ }^{11} \mathrm{C},{ }^{13} \mathrm{~N}$ and ${ }^{41} \mathrm{Ar}$, were observed when the ORELA was operating and not observed when the ORELA was shut off.

Gamma-ray assay measurements were obtained using a calibrated, highresolution, Ge detection system. Background data were obtained to ascertain quantitatively the sample-independent background contributions to the measurements. Data reduction utilized a combination of computer and manual methods. A complete analysis was carried out to determine the actual measured isotope radioactivity density (in $\mathrm{pCi} / \ell$ ) for the particular samples obtained and under the particular conditions existing when the samples were obtained.

Corrections were applied to these results to account for

(a) sample loss during transfer from the collection container to the Marinelli beaker,

(b) isotope decay characteristics (particularly lifetimes), and

(c) non-constant sample collection rate,

so as to present the final results as radioactivity density values.

The final results are collected in Table 5 . For comparison, results quoted ${ }^{1}$ for a sample drawn from the same exhaust stack at an earlier date are included. The comparisons can be only qualitative since the report on the earlier data states explicitly that these earlier data were not corrected for sample loss during transfer and the report does not indicate if the data were adjusted for variable-rate sample collection. In addition the ORELA operating conditions were somewhat different during the earlier measurement. The target room pressure was $45 \mathrm{~mm} \mathrm{Hg}$ compared to $25 \mathrm{~mm} \mathrm{Hg}$ in the present study. The ORELA power was $10 \mathrm{~kW}$ for the earlier measurement compared to $15 \mathrm{~kW}$ for the present measurement. Also, the Be-block target system was in use during the earlier measurement whereas the Ta target system was in use during the present study. This difference in target system means a difference in the created neutron flux distribution. Lastly, the sample counting $\mathrm{cu}_{2 .}$ itions were different, and, in particular, the earlier gamma-ray assay was not initiated soon enough to obtain data on the shorter-lived radionuclides of ${ }^{11} \mathrm{C}$ and ${ }^{13} \mathrm{~N}$. With these caveats, the only real difference between the present results and the earlier results is the positive report of a small amount of ${ }^{134} \mathrm{I}$ in the latter.

In the final column of Table 5 are given the (allowed) Derived Air Concentrations for the several radioisotopes applicable to air inhaled by workers at DOE facilities. ${ }^{2}$ Comparisons of the experimental results with the given $D A C$ values requires some care and observation. The first point is that the given DAC values are for whatever meteorological conditions are extant at the time of the exposure. The second point is that the present measurements are sampled from air which is being confined to the exhaust stack; no account for dispersal into the atmosphere following complete 
exhaust has been taken. Even so, the present results are more than a factor of 50 smaller than the DAC values for every radionuclide in Table 5.

Insofar as recommendations for follow-on activities, if the observed levels of emissions are found to be of significance for workers or members of the public, a regular schedule of stack-exhaust monitoring should be designated and followed. In addition, to this author it would seem prudent to conduct similar samplings for a variety of operating conditions. Such additional studies should aid in more accurately characterizing the sources of the observed radionuclides with the ultimate goal of reducing any significant radioisotope contributions to the atmosphere due to ORELA operations.

Table 5. Radionuclide activity in the Building 6010 exhaust gases (in $\mathrm{pCi} / \ell$ ) deduced by gamma-ray assay of cryogenic samples.

\begin{tabular}{lccr}
\hline Radionuclide & $\begin{array}{c}\text { Present } \\
\text { results }^{a}\end{array}$ & $\begin{array}{c}\text { Results of } \\
\text { June 2, } 1989^{b}\end{array}$ & $\begin{array}{c}\text { Air Immersion } \\
\text { DAC }^{c}\end{array}$ \\
\hline${ }^{11} \mathrm{C}$ & $18.2 \pm 4.2^{d}$ & & 4000 \\
${ }^{13} \mathrm{~N}$ & $73 \pm \pm 14$ & & 4000 \\
${ }^{41 \mathrm{Ar}}$ & $13.2 \pm 0.7$ & $20.2 \pm 1.3^{e}$ & 3000 \\
${ }^{222} \mathrm{Rn}$ & $0.62 \pm 0.25^{f}$ & $1.39 \pm 0.35$ & 80 \\
${ }^{133} \mathrm{~m} \mathrm{Xe}$ & $-0.12 \pm 0.59$ & $0.65 \pm 0.66$ & 100000 \\
${ }^{133} \mathrm{I}$ & $0.0 \pm 0.5$ & $0.17 \pm 0.16$ & 100 \\
${ }^{134} \mathrm{I}$ & $0.20 \pm 0.38$ & $1.67 \pm 0.05$ & 1000 \\
\hline
\end{tabular}

${ }^{a}$ All corrections included.

${ }^{b}$ Data from ref. 1. Transfer corrections not included.

c Derived Air Concentrations (DAC) for controlling radiation exposures to workers from ref. 2; value for ${ }^{133}$ I taken from page 12 , and the other values from pages 34-38.

${ }^{d}$ One-standard-deviation uncertainties for the present study.

e Two-standard-deviation uncertainties according to ref. 1 .

${ }^{f}$ Same amount observed in the ORELA-off sample as observed in the ORELAon sample. 


\section{REFERENCES}

1. D. B. Deizer, "Cryogenic Sampling of Stack 6010 and 3039 NE Duct," Memorandum to V. L. Turner (Martin Marietta Energy Systems, Inc., Internal Correspondence,unnumbered, June 6, 1989).

2. U.S. Department of Energy, "Radiation Protection for Occupational Workers," ORDER DOE 5480.11 (Dec. 21, 1988), Attachment 1.

3. Handbook of Chemistry and Physics, Ed. R. C. Weast, 52nd edition (Chemical Rubber Co., Cleveland, Ohio 44128, 1971), p. 166 and 1970.

4. C. E. Burgart, E. A. Straker, T. A. Love, and R. M. Frestone, Jr., Nucl. Sci. Engr. 42, 421 (1970).

5. G. L. Morgan, T. A. Love, and F. G. Perey, Nucl. Instrum. Meth. 128, 125 (1975).

6. All nuclear data (lifetimes and branching ratios) were taken from Table of Isotopes, eds. C. M. Lederer and V. S. Shirley (John Wiley and Sons, Inc., New York, 1978).

7. P. T. Perdue and F. F. Haywood, "Cryogenic Method for Measuring Nuclides and Fission Gases," U.S. Patent Application No. 145,643 (2 May 1980).

8. American Institute of Physics Handbook, eds. D. E. Gray, D. N. Fischel, H. B. Crawford, D. A. Douglas, and W. C. Eisler, third edition (McGraw-Hill, Inc., 1972), p. 2-161.

9. D. R. Kelsheimer and R. Sallie, "Demonstration of Noble Gas Collection Using the Cryogenic Technique," Martin Marietta Energy Systems, Inc., Oak Ridge Gaseous Diffusion Plant Report No. K/QT-222 (September 1988).

10. R. Sallie and D. B. Delzer, "Traceability of Results for Rare Gas Stack Monitoring Program," Martin Marietta Energy Systems, Inc., Oak Ridge Gaseous Diffusion Plant Report No. K/QT-299 (June 16, 1989).

11. Amersham International, Solution No. R8/125/14 calibrated as of $1200 \mathrm{GMT}$ on June 1, 1988.

12. National Bureau of Standards (Gaithersburg, MD 20899) Standard Reference Material 4276B, Source ID SRM 4276B-155, calibrated as of 1200 EST May 1, 1983.

13. J. K. Dickens, TPASS, A Gamma-ray Spectrum Analysis and Isotope Identification Computer Code, ORNL-5732 (1982).

14. "TPASGAM 85," RSIC Data Library Collection, DLC-88 (1985), distributed by the Radiation Shielding Information Center of the Oak Ridge National Laboratory, Oak Ridge, TN.

15. R. L. Bywater, Jr., Gamma-ray Production Cross Sections for 0.9 to $20 \mathrm{MeV}$ Neutron Interactions with ${ }^{10} \mathrm{~B}$, ORNL/TM-10191 (1986).

16. P. R. Bevington, Data Reduction and Error Analysis for the Physical Sciences (McGraw-Hill, New York, 1969), p. 72ff.

17. K. Masumoto, T. Kato, and N. Suzuki, Nucl. Instrum. Meth 157, 567 (1978). 
18. O. D. Brill', N. A. Vlasov, S. P. Kalinen, and L. S. Sokolov, Doklady Akad. Nauk, SSSR 136, 55 (1961); Eng. transl. Sov. Phys. Doklady 6, 24 (1961).

19. F. G. Perey, Oak Ridge National Laboratory Report ORNL/TM-6267, ENDF259, 1978, p. 26; J. K. Dickens and J. W. McConnell, Phys. Rev. C 23, 331 (1981).

20. P. R. Bevington, op. cit., p. 190. 

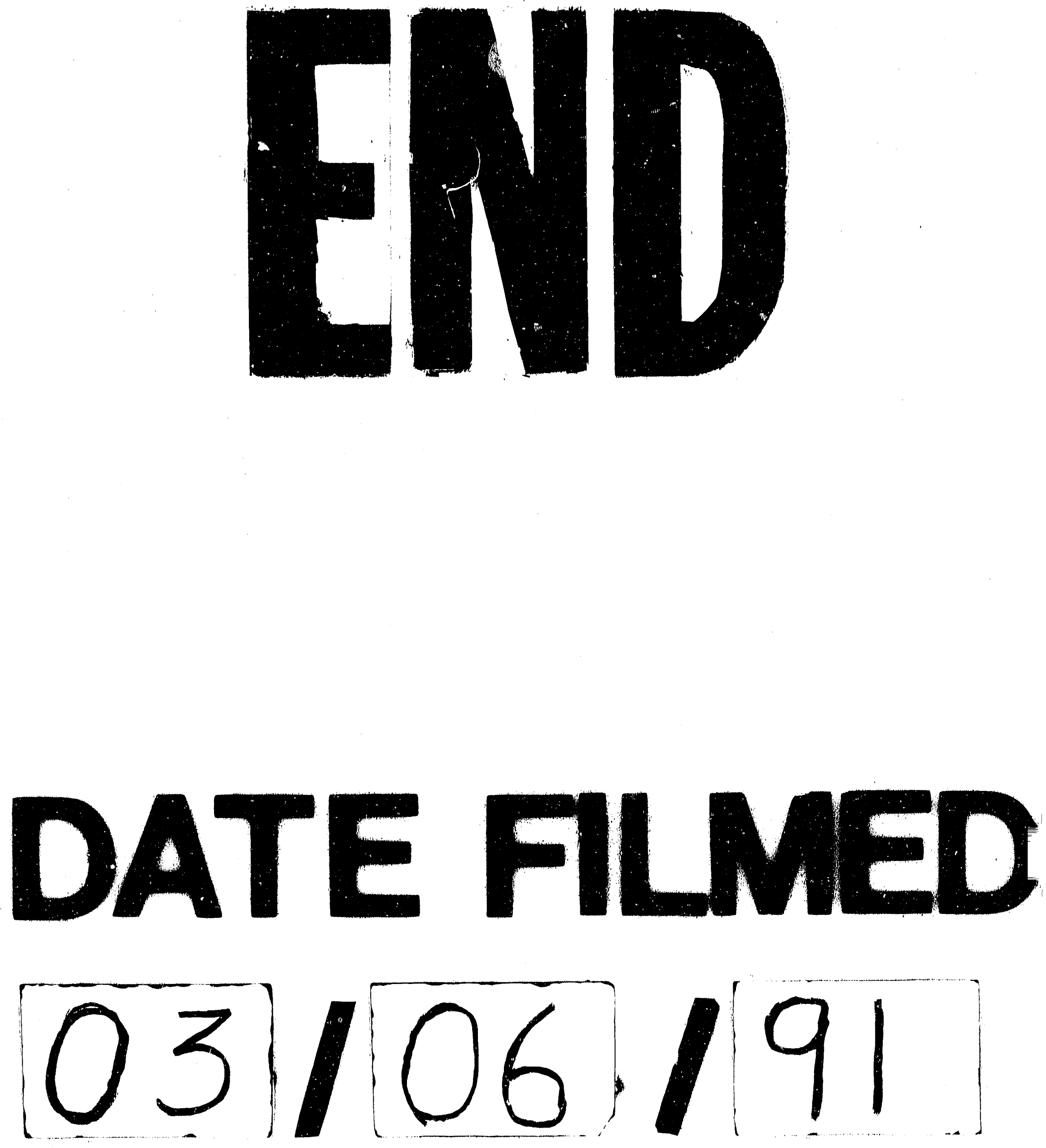
